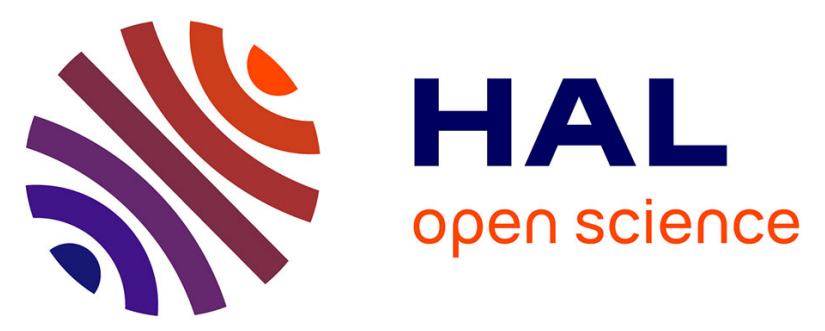

\title{
Recommandations françaises pour les critères diagnostiques d'arrêt de grossesse au premier trimestre et stratégie de prise en charge des patientes présentant une menace de fausse couche ou une grossesse arrêtée (hors évacuation utérine)
}

Vincent Lavoué, Cyrille Huchon, Xavier Deffieux, Marie-Catherine Voltzenlogel, Laurent Vandenbroucke, Jean Levêque

\section{- To cite this version:}

Vincent Lavoué, Cyrille Huchon, Xavier Deffieux, Marie-Catherine Voltzenlogel, Laurent Vandenbroucke, et al.. Recommandations françaises pour les critères diagnostiques d'arrêt de grossesse au premier trimestre et stratégie de prise en charge des patientes présentant une menace de fausse couche ou une grossesse arrêtée (hors évacuation utérine). Journal de Gynécologie Obstétrique et Biologie de la Reproduction, 2014, 43 (10), pp.776-793. 10.1016/j.jgyn.2014.09.012 . hal-01102771

\author{
HAL Id: hal-01102771 \\ https://hal.science/hal-01102771
}

Submitted on 13 Jan 2015

HAL is a multi-disciplinary open access archive for the deposit and dissemination of scientific research documents, whether they are published or not. The documents may come from teaching and research institutions in France or abroad, or from public or private research centers.
L'archive ouverte pluridisciplinaire HAL, est destinée au dépôt et à la diffusion de documents scientifiques de niveau recherche, publiés ou non, émanant des établissements d'enseignement et de recherche français ou étrangers, des laboratoires publics ou privés. 
Recommandations françaises pour les critères diagnostiques d'arrêt de grossesse au premier trimestre et stratégie de prise en charge des patientes présentant une menace de fausse couche ou une grossesse arrêtée (hors évacuation utérine)

French guidelines for diagnostic criteria for nonviable pregnancy early in the first trimester and for management of threatened miscarriage and nonviable pregnancy (except uterine evacuation)

Vincent Lavoué ${ }^{1}$ - Cyrille Huchon ${ }^{2}$ - Xavier Deffieux ${ }^{3}$ - Marie-Catherine Voltzenlogel ${ }^{1}-$ Laurent Vandenbroucke $^{1}$ - Jean Levêque ${ }^{1}$

1, Centre Hospitalier Universitaire de Rennes, Université de Rennes 1. INSERM U1085. Service de Gynécologie, Hôpital Sud, 16 boulevard de Bulgarie, 35000 Rennes, France.

${ }^{2}$, CHI Poissy-St-Germain, Université Versailles-Saint-Quentin en Yvelines, Service de gynécologie \& obstétrique, 10 rue du champ Gaillard BP 308278303 Poissy CEDEX, France

${ }^{3}$, AP-HP, Hôpital Antoine Béclère, Service de Gynécologie-Obstétrique et Médecine de la Reproduction, Clamart F-92141, France; Université Paris-Sud, Faculté de Médecine, Orsay F91405, France; GREEN GRC 01 (UPMC), Groupe de RecherchE cliniquE en NeuroUrologie, Site Antoine Béclère, Clamart F-92141, France

Correspondance : vincent.lavoue@chu-rennes.fr 


\section{Résumé :}

Objectifs : évaluer la valeur diagnostique des examens complémentaires pour affirmer l'arrêt d'une grossesse au premier trimestre de grossesse et la stratégie de prise en charge des patientes ayant une grossesse arrêtée au premier trimestre (hors évacuation).

Méthode: Recherche bibliographique en langues française et anglaise effectuée par consultation des banques de données PubMed, Cochrane Library et des recommandations des sociétés savantes internationales.

Résultats: En échographie endovaginale, une mesure échographique moyenne du sac gestationnel intra-utérin (mesurée sur 3 plans de coupe orthogonaux) $\geq 25 \mathrm{~mm}$ sans embryon (NP2) ou une longueur cranio-caudale d'un embryon mesurée dans le plan sagittal $\geq 7 \mathrm{~mm}$ sans activité cardiaque (NP2) permet d'affirmer l'arrêt d'une grossesse intra-utérine. En cas de grossesse intra-utérine d'évolutivité incertaine, un contrôle échographique réalisé à plus de 7 ou 14 jours en fonction de la présence ou non d'une vésicule vitelline ou d'un embryon à l'échographie endovaginale permet d'affirmer l'arrêt de la grossesse (NP4). En cas de grossesse de localisation indéterminée, une augmentation des hCG sériques $\leq 15 \%$ à 48 heures en cas de hCG initial $<2000 \mathrm{UI} / \mathrm{L}(\mathrm{NP} 2)$ ou une progestéronémie faible $(<3,2 \mathrm{ng} / \mathrm{mL})$ (NP2) excluent une grossesse intra-utérine évolutive. 


\begin{abstract}
:
Objective: To assess value of sonography, hCG and progesterone for diagnosis of firsttrimester non-viable pregnancy and to assess first-trimester miscarriage management (except evacuation).
\end{abstract}

Methods: French and English publications were searched using PubMed, Cochrane Library and international learned societies recommendations.

Results: First-trimester nonviable pregnancy is established with gestational sac mean diameter and embryo crown-rump length. Mean sac diameter $\geq 25 \mathrm{~mm}$ without embryo (LE2) or embryo crown-rump length $\geq 7 \mathrm{~mm}$ without heartbeat (LE2) by transvaginal sonography allows to diagnose pregnancy failure. Intrauterine pregnancy of uncertain viability is defined by intra-uterine gestational sac without embryo with heartbeat or without pregnancy diagnosis failure and requires a new transvaginal sonography. The delay for this second sonography depends on the aspect of intrauterine picture (i.e. presence of yolk sac or not, presence of embryo or not) (LE4). Human chorionic gonadotropin (hCG) and progesterone are useful for pregnancy of unknown location (i.e no gestational sac at transvaginal sonography): hCG ratio $<15 \%$ between two-day serum samples when first hCG is $<2000 \mathrm{UI} / \mathrm{mL}$ (LE2) or low serum progesterone level $(<3,2 \mathrm{ng} / \mathrm{mL}$ ) (LE2) exclude viable intrauterine pregnancy. There is not known prevention strategy for threatened miscarriage. 
Mots Clés: Grossesse arrêtée, fausse couche, métrorragie, échographie, beta-hCG, progestéronémie.

Keywords: Miscarriage, spontaneous abortion, sonography, human Chorionic Gonadotrophin, progesterone. 


\section{EQUATION DE RECHERCHE ET METHODE D’EVALUATION}

La recherche a été réalisée sur les 25 dernières années en utilisant les termes « MeSH ». Les sources suivantes ont été interrogées:

- Medline : PubMed (Internet portal of the National Library of Medicine)

http://www.ncbi.nlm.nih.gov/sites/entrez?db=pubmed

- $\quad$ The Cochrane library: Cochrane-database 'Cochrane Reviews' and 'Clinical Trials' http://www3.interscience.wiley.com/cgi-bin/ mrwhome/106568753/HOME

\section{$\underline{\text { DARE }}$}

- Agency for Healthcare Research and Quality, U.S. Department of Health and Human Services, 540 Gaither Road,Rockville, MD 20850

www.ahrq.gov

La recherche dans ces bases de données a été complétée par la revue des références contenues dans les méta-analyses, revues systématiques et articles originaux inclus. Seuls les articles publiés en langue anglaise ont été pris en compte.

\section{Equations de recherche:}

("spontaneous abortion"[MeSH Terms] $\quad$ OR $\quad$ ("miscarriage"[All Fields]) AND ("humans"[MeSH Terms] AND "female"[MeSH Terms] AND (Clinical Trial[ptyp] OR MetaAnalysis[ptyp] OR Practice Guideline[ptyp] OR Randomized Controlled Trial[ptyp] OR Comparative Study[ptyp] OR Controlled Clinical Trial[ptyp] OR Multicenter Study[ptyp]) AND (English[lang]) AND jsubsetaim[text] AND "1985/01/01"[PDat] : "2013/11/30"[PDat]). 


\section{Introduction}

Une grossesse symptomatique (métrorragies et/ou douleurs) au premier trimestre pose deux types de problèmes : la localisation de la grossesse et son évolutivité.

Valeur de l'examen clinique pour diagnostiquer une grossesse évolutive ou une fausse couche.

Dans une étude de cohorte prospective de 204 patientes présentant des métrorragies au premier trimestre de grossesse, l'utilisation du jugement clinique ou d'un score clinique (interrogatoire sur les saignements et examen au speculum) sans échographie par 70 médecins généralistes donnent un diagnostic erroné de grossesse évolutive ou de fausse couche complète dans respectivement 41 et $58 \%$ des cas (NP2) [1, 2]. La symptomatologie (métrorragies, douleurs pelviennes), l'anamnèse et l'examen gynécologique chez une femme enceinte au premier trimestre de grossesse sont donc moins sensibles et spécifiques pour le diagnostic de grossesse évolutive ou de fausse couche complète comparés à l'échographie, considéré comme l'examen gold standard (NP2) [1, 2].

Devant des métrorragies ou des douleurs pelviennes en début de grossesse, une échographie pelvienne est recommandée pour permettre le diagnostic de localisation et d'évolutivité de la grossesse (grade B).

Premières structures identifiables à l'échographie endovaginale d'une grossesse Sac gestationnel

Plusieurs études prospectives portant sur 38 à 361 patientes [3-6] ont évalué par échographie endovaginale l'apparition du premier signe visible de grossesse intra-utérine (GIU). Ce premier signe visible est le sac gestationnel correspondant à une image anéchogène ronde entourée par un anneau échogène au sein de la réaction déciduale de l'endomètre près du fond 
utérin (NP2) (figure 1). Ce sac gestationnel apparaît entre le $28^{\text {ème }}$ et le $31^{\text {ème }}$ jour. A 32 jours d'aménorrhée, en cas de grossesse évolutive, le sac gestationnel est toujours visible [4].

\section{Vésicule Vitelline}

Plusieurs études prospectives portant sur 38 à 361 patientes [3-7] ont aussi évalué par échographie endovaginale l'apparition du deuxième signe visible de GIU. Le deuxième signe visible est la vésicule vitelline qui correspond à un anneau échogène excentré dans le sac gestationnel (NP2) (figure 1). La vésicule vitelline est visible au plus tôt à 35 jours d'aménorrhée (5 SA) et est vue pour la première fois chez toutes les patientes entre 36 et 40 jours d'aménorrhée (entre $5 \mathrm{SA}+1 \mathrm{j}$ et $5 \mathrm{SA}+5$ jours) (NP2) [4]. La vésicule vitelline augmente de taille de 3 à $5 \mathrm{~mm}$ jusqu'à $9 \mathrm{SA}$ pour ensuite régresser et disparaître à partir de $12 \mathrm{SA}$ (NP3) [7].

\section{Embryon}

Plusieurs études prospectives portant sur 38 à 426 patientes [3-8] ont évalué par échographie endovaginale l'apparition du troisième signe visible de GIU. Le 3ème signe visible est le pôle embryonnaire qui correspond à une structure linéaire échogène à la périphérie de la vésicule vitelline et mesurant 1 à $2 \mathrm{~mm}$ à 35 jours d'aménorrhée (NP2) (figure 1). La croissance embryonnaire a été évaluée pour la première fois par échographie sus-pubienne en 1972 pour établir les courbes de croissance dites de Robinson $[9,10]$. Compte tenu du fait que l'échographie endovaginale est la voie échographique préférentielle (cf plus loin) pour l'échographie d'une grossesse avant 8 ou 10 SA, ce sont les courbes établies en 1992 par Hadlock et al qui sont les plus pertinentes [11]. Hadlock et al ont établi la croissance de l'embryon par échographie endovaginale jusqu'à 8SA et par échographie endovaginale et suspubienne entre 8 et $13 \mathrm{SA}$ en fonction du terme dans une série prospective de 416 patientes américaines (NP3) [11]. Ces données ont été confirmées par 3 études prospectives effectuées par échographie endovaginale dans les années 1990 chez 94 patientes prises en charge en FIV 
[12], 160 patientes prises en charge également en assistance médicale à la procréation [13] et 270 patientes avec une grossesse spontanée et datée de manière certaine [14]. Dans ces études, il existe une variation de la longueur cranio-caudale (LCC) pour un même âge gestationnel estimé à 8\% (2 SD) [11]. L'embryon augmente de taille autour de $1 \mathrm{~mm}$ par jour [15]. Entre 6 et $8 \mathrm{SA}$, l'embryon mesure de 4 à $18 \mathrm{~mm}$ et, entre 8 et $10 \mathrm{SA}$, l'embryon mesure de 15 à $30 \mathrm{~mm}[11,15]$ (NP1). Une étude rétrospective de 2010 portant sur 3710 grossesses a réévalué les courbes de croissance de l'embryon en fonction du terme gestationnel (NP4) [16]. Cette étude a montré une différence de $2,7 \mathrm{~mm}$ à $6 \mathrm{SA}$ entre ces nouvelles courbes et les courbes de Hadlock, soit une sous estimation de 3 jours en terme d'âge gestationnel pour les courbes de Hadlock. A 9 SA, les courbes se superposent. A 14 SA, il existe une différence de 4,8 mm entre les deux courbes, soit une surestimation de 2 jours de la courbe de Hadlock. Cependant, cette dernière étude de 2010 était rétrospective (soit un niveau de preuve inférieur aux données précédemment publiées qui étaient prospectives). On retiendra donc les valeurs des courbes d'Hadlock, en l'absence de nouvelle étude prospective. (Tableau 1)

\section{Activité Cardiaque}

L'activité cardiaque est visualisée sous forme d'un clignotement au niveau du pôle embryonnaire en mode $B$ et peut également être visualisée en mode $M$ [8]. L'activité cardiaque embryonnaire est visualisée en échographie endovaginale à 35 jours d'aménorrhée dans $15 \%$ des grossesses $(n=15)$, à 36 jours d'aménorrhée dans $82 \%$ des grossesses $(n=19)$ et après 37 à 46 jours d'aménorrhée dans $100 \%$ des grossesses évolutives sur des séries prospectives de 27 à 137 patientes (NP2) [4, 8, 18]. Une étude prospective portant sur 137 patientes entre 5 et 12 semaines d'aménorrhée a montré que l'activité cardiaque embryonnaire était observée par échographie endovaginale après 46 jours (soit une LCC $>6 \mathrm{~mm}$ ) d'aménorrhée avec une sensibilité de 100\%, une spécificité de 93,1\%, une valeur prédictive positive de $96,9 \%$ et une valeur prédictive négative de 100\% (NP2) [18]. 
Techniques de mesure échographique du sac gestationnel, de la vésicule vitelline et de l'embryon

Plusieurs techniques de mesure concernant le sac gestationnel intra-utérin ont été proposées. L'équipe de Coulam et coll réalisent la mesure du sac gestationnel par la moyenne de deux mesures : une mesure transversale et une mesure longitudinale sur leur série prospective de 361 patientes ou leur série cas témoins de 67 patientes $[6,8,19]$. Ce groupe ne précise pas la position des calipers. Dans les études publiées après 2000, la mesure du sac gestationnel est toujours réalisée par la moyenne de trois mesures orthogonales ( 2 dans le plan sagittal et 1 dans le plan transversal) en plaçant les calipers sur le bord interne du sac gestationnel, soit à la limite externe de la zone anéchogène du sac gestationnel, en échographie endovaginale (figure 2) [2, 15, 20-24].

La mesure de la vésicule vitelline a été réalisée par la moyenne de deux mesures (une transversale et une longitudinale) du diamètre de la vésicule vitelline dans l'étude prospective de 180 patientes de l'évolution de la taille de la vésicule vitelline au premier trimestre de grossesse de Jauniaux et coll [7]. Cette étude n'a pas précisé la position des calipers lors de l'échographie. Dans la revue de la littérature portant sur la croissance de la grossesse au premier trimestre de grossesse, Bottomley $\mathrm{C}$ et Bourne $\mathrm{T}$ indiquent que la mesure de la vésicule vitelline doit être faite par la moyenne de la mesure de trois plans orthogonaux en plaçant les calipers de mesure sur la bordure externe de la vésicule vitelline, sans citer les articles de référence [15]. Dans la plupart des études, seule la présence ou l'absence de vésicule vitelline est notée, sans préjuger de sa taille [20].

Pour la technique de mesure de l'embryon, nous n'avons retenu que les études pour lesquelles la technique de mesure est correctement décrite avec notamment la position des calipers. Dans l'étude rétrospective de cohorte de Pexsters et al portant sur 3710 grossesses pour 
l'évaluation de la mesure cranio-caudale de l'embryon par échographie endovaginale [16], la technique de mesure a été réalisée en plaçant les calipers de mesure sur le bord externe de la partie craniale et de la partie caudale de l'embryon dans cette étude [16]. Dans l'étude l'étude prospective de la variabilité inter et intra-observateur des mesures embryonnaires portant sur 54 patientes, la même technique de mesure a été réalisée [25] (NP2). La mesure de l'embryon doit être la longueur cranio-caudale en coupe sagittale, en plaçant les calipers de mesure sur le bord externe de la partie craniale et de la partie caudale de l'embryon, en échographie endovaginale (figure 3). 
Quelle est la variabilité intra et inter-observateur pour la mesure échographique de la longueur cranio-caudale de l'embryon et du diamètre du sac gestationnel ?

Une seule étude de cohorte transversale prospective non consécutive a évalué la variabilité intra-opérateur et inter-opérateur de la mesure de la longueur cranio-caudale de l'embryon et du sac gestationnel dans les GIU précoces (de 6 à 9 semaines d'aménorrhée) par voie échographique endo-vaginale (sonde 6-12Mhz, mode B). Elle a évalué la reproductibilité des mesures entre 2 opérateurs sur 44 patientes et la reproductibilité intra-opérateur sur 54 patientes de manière prospectives [25]. Cette étude a montré une très bonne reproductibilité avec un coefficient de corrélation interclasse à 0,992 pour la variabilité inter et intra opérateur de la mesure de la LCC et de 0,952 pour la variabilité inter et intra opérateur de la mesure du sac gestationnel. Le calcul des limites de concordance à 95\% de Bland et Altman a montré une variabilité intra-opérateur pour la longueur craniocaudale de l'embryon de $\pm 8,91 \%$ à $\pm 11,37 \%$ et inter-opérateur de $\pm 14,64 \%$ (NP3). Elle a également montré une variabilité interopérateur pour la mesure du sac gestationnel de $\pm 18,78 \%$ (NP3). Une seconde étude de cohorte transversale prospective non consécutive a évalué la variabilité intra-opérateur et inter-opérateur de la mesure de la longueur cranio-caudale de l'embryon dans les GIU de 9 à 14 semaines d'aménorrhée par voie échographique abdominale (sonde courbe de 5Mhz). Elle a évalué la reproductibilité de mesures entre 2 opérateurs sur 21 patientes [26]. Cette étude a montré une très bonne reproductibilité avec un coefficient de corrélation interclasse à 0,995 pour la variabilité inter-opérateur et de 0,998 pour la variabilité intra-opérateur pour la mesure de la LCC. Cette étude a calculé les limites de concordance à 95\% de Bland et Altman et montré une variabilité intra-opérateur pour la longueur craniocaudale de l'embryon de $\pm 2,7 \%$ à $\pm 2,3 \%$ et inter-opérateur de $\pm 6,1$ à $\pm 5,7 \%$ (NP3). Cette étude a montré que plus la mesure de la longueur cranio-caudale embryonnaire était petite plus la variabilité était grande, sans qu'il 
y ait un seuil identifié de la longueur craniocaudale à partir duquel la reproductibilité était faible. Le moment optimal pour dater une grossesse de manière précise et reproductible est quand la croissance de l'embryon ou du foetus est la plus importante, avec peu de variabilité, et avant les mouvements de flexion ou d'extension qui induisent des erreurs de mesure. Ce moment optimal est potentiellement entre 8 et $12 \mathrm{SA}$, sans qu'aucune étude ne permette de l'affirmer [15].

La variabilité inter et intra-observateur de la mesure du sac gestationnel et de la longueur cranio-caudale d'une grossesse intra-utérine précoce par échographie endovaginale est faible et est estimée respectivement à $\pm 8,91 \%$ et $\pm 18,78 \%$ au maximum (NP3). 
Quelle est la voie d'abord échographique optimale (endovaginale ou sus-pubienne) pour la prise en charge d'une patiente présentant des douleurs ou des métrorragies au premier trimestre de grossesse?

Une étude de cohorte transversale non consécutive publiée en 1993 est disponible comparant les performances diagnostiques de l'échographie endovaginale aux performances de l'échographie sus-pubienne dans le diagnostic d'une GIU arrêtée au premier trimestre de grossesse. Cette étude a comparé 290 patientes référées à une consultation de génétique avec une échographie sus-pubienne à 308 patientes référées à une consultation de demande d'interruption volontaire de grossesse avec une échographie endo-vaginale (NP4) [27]. L'échographie réalisée évaluait la taille du sac gestationnel, de l'embryon (longueur craniocaudale) et l'activité cardiaque. Les taux de détection du sac gestationnel, de l'embryon et de l'activité cardiaque sont significativement meilleurs jusqu'à 39 jours d'aménorrhée (soit 5 semaines d'aménorrhée et demi) par échographie endovaginale $(p<0,0001)$ (NP4). L'âge gestationnel du diagnostic de GIU arrêtée n'est pas significativement différent dans les deux séries.

Une autre étude de cohorte consécutive publiée en 1988 a comparé les performances de l'échographie endovaginale à l'échographie sus-pubienne dans une population de 90 patientes enceintes symptomatiques sous forme de douleurs et/ou de métrorragies [28]. Sur ces 90 patientes, 55 patientes avaient au final une GIU normale, 22 une grossesse ectopique, 7 une grossesse arrêtée et 6 une fausse couche complète. Les 55 (100\%) GIU évolutives ont été repérées par échographie endovaginale contre 11 (20\%) par échographie sus pubienne. La vésicule vitelline, le pôle embryonnaire et l'activité cardiaque ont été identifiés dans les 34 premiers jours d'aménorrhée par voie endovaginale contre les 42 premiers jours d'aménorrhée pour la voie sus-pubienne. L'échographie endovaginale permet de détecter plus précocement l'apparition des différentes structures embryonnaires (sac 
gestationnel, vésicule vitelline et pôle embryonnaire) que l'échographie sus-pubienne (NP4). Parmi les 22 grossesses ectopiques, l'échographie endovaginale a pu détecter le sac gestationnel extra-utérin dans 3 cas contre 0 pour l'échographie sus-pubienne. Pour les 6 cas de fausse couche spontanée, l'échographie endovaginale a pu détecter les 6 contre seulement 3 pour l'échographie sus pubienne (NP4). Une étude de cohorte prospective consécutive publiée en 1991 portant sur 175 patientes avec une grossesse évolutive a comparé les performances de l'échographie endovaginale aux performances de l'échographie suspubienne pour la détection de l'activité cardiaque embryonnaire en fonction de la longueur cranio-caudale [29]. L'échographie endovaginale permet de détecter une activité cardiaque dans $100 \%$ des cas pour une longueur cranio-caudale embryonnaire de plus de $5 \mathrm{~mm}$ contre 9 mm par voie sus-pubienne. L'échographie endovaginale permet donc une détection plus précoce de l'activité cardiaque d'une GIU que l'échographie sus-pubienne (NP2). Dans le cadre plus large des pathologies du premier trimestre de grossesse incluant aussi les GEU en plus des GIU, une étude de cohorte de 100 patientes consécutives symptomatiques avec une grossesse de localisation indéterminée à l'échographie suspubienne (sonde de 3,5 à 5 Mhz) a étudié l'apport de l'échographie endo-vaginale (sonde de $5 \mathrm{MHz}$ ) pour permettre la localisation de la grossesse [30] (NP2). L’échographie endovaginale a modifié le diagnostic de localisation apporté par l'échographie sus-pubienne et permis de localiser la grossesse pour 44\% des patientes (31\% des GEU et 52\% des GIU). L'échographie endovaginale augmente donc le taux de localisation d'une grossesse en cas de grossesse de localisation indéterminée diagnostiquée à l'échographie sus-pubienne (NP2).

En cas de doute sur la localisation ou l'évolutivité d'une grossesse à l'échographie suspubienne, la réalisation d'une échographie endovaginale est recommandée (grade B). 


\section{Quelles sont les caractéristiques échographiques nécessaires pour affirmer l'existence d'une GIU en l'absence d'embryon visualisé en intra-utérin ?}

La présence d'un embryon à l'intérieur d'une image hypoéchogène intra-utérine signe la GIU.

Deux signes ont été évalués à l'échographie en l'absence de vésicule vitelline ou d'embryon pour orienter vers le diagnostic de GIU. Le premier est le double cercle du sac («double decidual sac ») qui correspond à une couronne hyper-échogène interne (censé être le trophoblaste) et une couronne hypo-échogène externe (censé être la transformation gravidique de l'endomètre) décrit par Bradley et al en 1982 [31]. Une étude de cohorte associant 61 cas consécutifs prospectifs de suspicion de GEU et 17 cas non consécutifs rétrospectifs a étudié ce signe et retrouvé une spécificité de $100 \%$ pour le diagnostic de GIU (NP4) [31]. Le deuxième signe est la couronne trophoblastique («intradecidual sign») qui présente une forte échogénicité décrit par Yeh et al en 1986 [32]. "L'intradecidual sign » a été évalué par voie endovaginale sur une cohorte prospective de 102 patientes présentant une image intrautérine sans vésicule vitelline ou embryon par 4 opérateurs indépendants [33]. La sensibilité de ce signe quant au diagnostic de GIU était de $34-66 \%$ et la spécificité de 55 à $73 \%(\mathrm{NP} 4)$. Une étude de cohorte non consécutive rétrospective de 187 patientes présentant une image anéchogène intra-utérine sans vésicule vitelline a été également réalisée pour évaluer «l'intradecidual sign » pour le diagnostic de GIU [34]. La sensibilité de ce signe quant au diagnostic de GIU était de 60-68\% et la spécificité de 97-100\% (NP4). Une étude rétrospective de cohorte sur 199 échographies endovaginales de patientes présentant une image intra-utérine anéchogène sans vésicule vitelline, ni embryon et dont le suivi a prouvé l'existence d'une GIU, a évalué la variabilité inter-observateur de "l'intradecidual sign » et du signe du «double decidual sac» [35] (NP4). La reproductibilité inter-observateur était faible pour les 2 signes $(\kappa=0,23)(\mathrm{NP} 4)$. Ces deux signes sont notamment absents dans au moins $35 \%$ des sacs gestationnels sans vésicule vitelline dont la nature intra-utérine a été 
ensuite validée [35] (NP4). Ainsi, une structure ronde anéchogène intra-utérine chez une patiente avec des beta-hCG positifs est probablement une GIU et moins probablement un pseudo-sac gestationnel, qui peut être retrouvé en cas de grossesse extra-utérine [36].

Aucune étude n'a été retrouvée évaluant la sensibilité, la spécificité, les valeurs prédictives négatives et positives de la visualisation de la vésicule vitelline pour le diagnostic de GIU. Seules des études de cohorte prospectives portant sur 38, 53, 170 et 361 patientes et évaluant l'apparition des premiers signes échographiques d'une GIU ont montré que l'apparition de la vésicule vitelline était rapidement suivie de l'apparition d'un embryon, signant la GIU [3-7] (NP4).

La présence d'un sac gestationnel avec une vésicule vitelline ou un embryon dans la cavité intra-utérine signe le diagnostic d'une GIU (NP4) et écarte le diagnostic de GEU, excepté dans les circonstances exceptionnelles comme la procréation médicalement assistée où il peut exister une grossesse hétérotopique [37, 38].

Les signes du «double decidual sac» et de «intradecidual sac» en échographie sont à la fois peu sensibles et peu spécifiques pour affirmer le diagnostic de GIU (NP4).

La mise en évidence d'un signe du «double decidual sac » ou du signe " intradecidual $s a c »$ en échographie n'est pas suffisante pour affirmer le diagnostic de GIU (NP4).

La présence d'un sac gestationnel sans embryon mais avec une image de vésicule vitelline à l'intérieur du sac gestationnel à l'échographie affirme la présence d'une GIU (NP4). 
En l'absence d'embryon visible, quelle est la taille échographique du sac gestationnel permettant d'affirmer une GIU arrêtée?

Une revue systématique de la littérature publiée en 2011 [39] a recensé 8 études de cohorte rétrospectives ou prospectives étudiant la taille du sac gestationnel pour déterminer le seuil permettant d'affirmer le diagnostic de GIU arrêtée (NP3). Les effectifs de ces études étaient de 55 à 211 patientes, regroupant 872 patientes au total. Les seuils retenus s'étalaient de 8 à $26 \mathrm{~mm}$ avec ou sans vésicule vitelline dans les différentes études, mais seulement deux études avaient une spécificité dont la borne inférieure de leur intervalle de confiance à $95 \%$ était supérieure à 95\%. En regroupant l'ensemble des effectifs, cette méta-analyse a défini une mesure moyenne du sac gestationnel sans embryon avec ou sans vésicule vitelline $\geq 25$ mm donnant une spécificité pour le diagnostic de grossesse arrêtée de $100 \%$ (Intervalle de confiance à $95 \%: 96 \%$ à $100 \%$ ) (NP3). La conclusion de cette revue systématique indiquait le manque d'étude récente de haute qualité, prospective pour le diagnostic de grossesse arrêtée.

Une grande étude de cohorte prospective multicentrique publiée en 2011 et portant sur 1060 patientes, consultant dans une « early pregnancy clinic », a étudié la taille du sac gestationnel intra-utérin sans embryon avec ou sans vésicule vitelline pour déterminer le seuil à partir duquel une GIU peut être affirmée arrêtée de manière certaine [20] (NP2). La taille du sac gestationnel était mesurée dans les 3 plans de coupe par échographie endovaginale (sonde 6$12 \mathrm{Mhz}$ ) et la moyenne de ces trois mesures donnait la taille moyenne du sac gestationnel. En l'absence d'embryon, avec ou sans vésicule vitelline, une taille moyenne de sac gestationnel $\geq$ $21 \mathrm{~mm}$ donne une spécificité de $100 \%$ pour le diagnostic de grossesse arrêtée (NP2). Compte tenu de la variabilité inter-opérateur et intra-opérateur estimée à $\pm 18,8 \%$ [25], par extrapolation une mesure à $20 \mathrm{~mm}$ peut varier lors d'un autre examen échographique entre 16,8 et $24,5 \mathrm{~mm}$. En prenant en compte cette variabilité, par extrapolation, un seuil relevé $\geq$ 
$25 \mathrm{~mm}$ pour la taille moyenne du sac gestationnel sans embryon avec ou sans vésicule vitelline donne une certitude quant au diagnostic de grossesse arrêtée et permet donc de minimiser le risque de faux positif dans le diagnostic de grossesse arrêtée. On peut conserver le même niveau de preuve (NP2) avec un seuil à $25 \mathrm{~mm}$ que pour le seuil à $21 \mathrm{~mm}$, car plus on augmente la taille du sac gestationnel sans image d'embryon avec ou sans vésicule vitelline à l'échographie endovaginale, plus augmente la spécificité du diagnostic de GIU arrêtée et cette spécificité est déjà de $100 \%$ pour $21 \mathrm{~mm}$.

On recommande l'utilisation d'un seuil à $25 \mathrm{~mm}$ pour la mesure moyenne du sac gestationnel intra-utérin (mesurée sur 3 plans de coupe orthogonaux en échographie endovaginale) sans embryon, avec ou sans vésicule vitelline, à partir duquel on peut affirmer l'arrêt d'une grossesse (Grade B). 
Quelle est la longueur cranio-caudale échographique d'un embryon sans activité cardiaque permettant d'affirmer l'arrêt de la grossesse ?

Une revue de la littérature publiée en 2011 [39] a recensé 8 études de cohorte rétrospectives ou prospectives étudiant la taille de l'embryon sans activité cardiaque permettant d'affirmer le diagnostic de GIU arrêtée (NP3). Les effectifs de ces études étaient de 55 à 211 patientes, regroupant ainsi 872 patientes. Les seuils retenus s'étalaient de 4 à $6 \mathrm{~mm}$, mais seulement deux études avaient une spécificité dont la borne inférieure de leur intervalle de confiance à 95\% était supérieure à 95\%. La conclusion de cette revue systématique indiquait le manque d'étude récente de haute qualité, prospective pour le diagnostic de grossesse arrêtée.

Une grande étude de cohorte prospective multicentrique portant sur 1060 patientes, consultant dans une «early pregnancy clinic», a étudié la longueur cranio-caudale de l'embryon sans activité cardiaque à partir de laquelle une GIU peut être affirmée arrêtée de manière certaine [20] (NP2). La longueur cranio-caudale était mesurée dans le plan sagittal par échographie endovaginale (sonde 6-12 Mhz). Une longueur cranio-caudale de l'embryon $\geq 5,3 \mathrm{~mm}$ donnait une spécificité de $100 \%$ pour le diagnostic de grossesse arrêtée. Par extrapolation, compte tenu de la variabilité inter-observateur et intra-opérateur estimée de $\pm 8,91 \%$ à $\pm 14,64 \%$ [25], une mesure de la longueur cranio-caudale embryonnaire à $6 \mathrm{~mm}$ peut varier lors d'un autre examen échographique entre 5,4 et 6,7 $\mathrm{mm}$. Par extrapolation, en prenant en compte cette variabilité, un seuil relevé $\geq 7 \mathrm{~mm}$ pour la longueur cranio-caudale en échographie endo-vaginale d'un embryon sans activité cardiaque donne une certitude quant au diagnostic de grossesse arrêtée et permet donc de minimiser le risque de faux positif dans le diagnostic de grossesse arrêtée. On peut conserver le même niveau de preuve (NP2) avec un seuil à $7 \mathrm{~mm}$ que pour le seuil à $5,3 \mathrm{~mm}$, car plus on augmente la mesure de la longueur cranio-caudale sans activité cardiaque à l'échographie endovaginale, 
plus augmente la spécificité du diagnostic de GIU arrêtée et cette spécificité est déjà de 100\% pour $5,3 \mathrm{~mm}$.

Ce seuil de longueur cranio-caudale de $7 \mathrm{~mm}$ a été définie par échographie vaginale et n'a pas été évalué par voie sus-pubienne. Un seuil de longueur cranio-caudale sans activité cardiaque $\geq 15 \mathrm{~mm}$ en cas d'échographie seulement réalisée par voie sus-pubienne pour définir une GIU arrêtée a été indiqué dans une revue de la littérature sans qu'il n'ait été possible de retrouver le niveau de preuve rattaché à ce chiffre [2].

On recommande l'utilisation d'un seuil à $7 \mathrm{~mm}$ pour la longueur cranio-caudale d'un embryon (mesuré dans le plan sagittal en échographie endovaginale) sans activité cardiaque à partir duquel on peut affirmer l'arrêt d'une grossesse (grade B). 
Définition d'une grossesse intra-utérine dont l'évolutivité est incertaine.

Compte tenu de la chronologie d'apparition des différentes structures embryonnaires à l'échographie endovaginale, en fonction du moment auquel est fait l'échographie, il existe des images échographiques pour lesquelles on ne peut ni affirmer une grossesse évolutive (absence d'activité cardiaque), ni affirmer une grossesse arrêtée (la moyenne de la mesure des trois diamètres du sac gestationnel est $<25 \mathrm{~mm}$ ou la longueur cranio-caudale embryonnaire est $<7 \mathrm{~mm}$, en échographie endovaginale). Ce concept de GIU d'évolutivité incertaine a été introduit par une revue de la littérature publiée en 2013 dans le New England Journal of Medicine [2] (tableau 2) (figure 4).

Chez une femme enceinte, la GIU d'évolutivité incertaine est définie à l'échographie endovaginale par l'exclusion :

- d'une grossesse évolutive (embryon avec présence d'une activité cardiaque)

ET

- d'une grossesse arrêtée (sac gestationnel $\geq 25 \mathrm{~mm}$ sans embryon par échographie endovaginale ou longueur de l'embryon $\geq 7 \mathrm{~mm}$ sans activité cardiaque par échographie endovaginale)

La GIU d'évolution incertaine correspond à une image échographique (par voie endovaginale) montrant un sac gestationnel $<25 \mathrm{~mm}$ sans embryon ou une image d'embryon $<7$ mm sans activité cardiaque. 


\section{Quel est le délai minimal pour réaliser une nouvelle échographie pelvienne pour permettre le diagnostic de GIU arrêtée ou de GIU évolutive en cas de GIU d'évolutivité incertaine ?}

Le diagnostic de GIU d'évolutivité incertaine est un diagnostic transitoire qui doit ensuite être suivi d'un diagnostic soit de grossesse arrêtée, soit de grossesse évolutive. En cas de GIU d'évolutivité incertaine à l'échographie chez une femme enceinte présentant des métrorragies ou des douleurs, la probabilité médiane estimée d'avoir une grossesse intra-utérine évolutive est de $26,8 \%$ [40]. Il n'y a pas eu d'étude comparative pour essayer de définir quel est le meilleur examen complémentaire (dosage sérique ? échographie ?...) à réaliser pour permettre de faire évoluer le diagnostic de grossesse d'évolutivité incertaine en diagnostic de grossesse arrêtée ou de grossesse évolutive.

Une nouvelle échographie endovaginale peut être réalisée suite au diagnostic de grossesse d'évolution incertaine. Il n'existe pas d'étude comparative permettant de définir le délai optimal minimal à respecter pour réaliser une nouvelle échographie permettant d'affirmer la grossesse arrêtée. Cependant des données existent permettant de définir un délai à respecter pour affirmer une grossesse arrêtée en fonction de l'image de grossesse observée à l'échographie endovaginale. Ces données sont issues des séries échographiques qui étudient les chronologies d'apparition des différentes structures de la grossesse : sac gestationnel, vésicule vitelline, pôle embryonnaire et activité cardiaque. Ces études portaient sur 38, 53, 97, 170, 361 et 426 patientes [3-8]. La cinétique d'apparition des différentes structures est un sac gestationnel puis une vésicule vitelline puis un embryon avec activité cardiaque [3-8]. Le sac gestationnel apparaît entre le $28^{\text {ème }}$ et le $31^{\text {ème }}$ jour dans 3 séries prospectives de 38,53 et 97 patientes [3-5] (NP2). A 32 jours d'aménorrhée, en cas de grossesse évolutive, le sac gestationnel est toujours visible chez les 53 patientes évaluées par Bree et coll [4] (NP2). 
Cette apparition du sac gestationnel est donc variable à 3 jours près par rapport à la date des dernières règles, soit $1 / 2$ semaine (NP4). La vésicule vitelline est visible au plus tôt à 35 jours d'aménorrhée (5 SA) et est vue pour la première fois chez toutes les patientes entre 36 et 40 jours d'aménorrhée (entre $5 \mathrm{SA}+1 \mathrm{j}$ et $5 \mathrm{SA}+5$ jours) [4]. Cette apparition de la vésicule vitelline est donc variable à 5 jours près par rapport à la date des dernières règles, soit $\pm 1 / 2$ semaine (NP4). Le pôle embryonnaire est visible au plus tôt à 35 jours d'aménorrhée [3-8] (NP2). Enfin pour Bree RL et coll dans une étude prospective portant sur 53 patientes évaluées par échographie endovaginale et avec une grossesse prouvée d'évolution normale par la suite, l'activité cardiaque est toujours vue après 40 jours d'aménorrhée [4]. Une seconde étude prospective en 1991 portant sur 137 patientes entre 5 et 12 semaines d'aménorrhée montre que l'activité cardiaque embryonnaire est observée après 6 semaines d'aménorrhée et 4 jours (soit 46 jours d'aménorrhée) avec une sensibilité de 100\%, une spécificité de 93,1\%, une valeur prédictive positive de $96,9 \%$ et une valeur prédictive négative de $100 \%$ [18] (NP2). Cette apparition de l'activité cardiaque est donc variable à 5 jours près par rapport à la date des dernières règles, soit $\pm \frac{1}{2}$ semaine (NP4).

Ainsi, par extrapolation, en tenant compte de la variabilité de $\pm 1 / 2$ semaine de la visualisation échographique des structures (vésicule, embryon et activité cardiaque) et des abaques d'apparition des différentes structures de la grossesse et de leur croissance à l'échographie endovaginale (tableau 1) (figure 5) :

- devant une GIU d'évolutivité incertaine présentant un sac gestationnel intra-utérin sans vésicule vitelline, un second contrôle échographique à au moins 14 jours montrant l'absence d'embryon avec activité cardiaque permet d'affirmer l'arrêt de la grossesse (NP4).

- devant une GIU d'évolutivité incertaine présentant un sac gestationnel intra-utérin avec vésicule vitelline mais sans embryon visible, un second contrôle échographique à 
au moins 11 jours montrant l'absence d'embryon avec activité cardiaque permet d'affirmer l'arrêt de la grossesse (NP4).

- devant une GIU d'évolutivité incertaine présentant un embryon de moins de $7 \mathrm{~mm}$ sans activité cardiaque, un second contrôle échographique à au moins 7 jours montrant l'absence d'activité cardiaque permet d'affirmer l'arrêt de la grossesse (NP4) [2].

En cas de GIU d'évolutivité incertaine présentant un sac gestationnel (i.e $<25 \mathrm{~mm}$, moyenne de 3 mesures orthogonales à l'échographie endovaginale) sans vésicule vitelline, on recommande l'utilisation d'un délai d'au moins 14 jours pour affirmer l'arrêt de grossesse, en l'absence d'apparition d'un embryon avec activité cardiaque à l'échographie (grade C).

En cas de GIU d'évolutivité incertaine présentant un sac gestationnel (i.e $<25 \mathrm{~mm}$, moyenne de 3 mesures orthogonales à l'échographie endovaginale) avec vésicule vitelline mais sans embryon visible, on recommande l'utilisation d'un délai d'au moins 11 jours pour affirmer l'arrêt de grossesse, en l'absence d'apparition d'un embryon avec activité cardiaque à l'échographie (grade C).

En cas de GIU d'évolutivité incertaine présentant un embryon de moins de $7 \mathbf{m m}$ à l'échographie endovaginale, on recommande l'utilisation d'un délai d'au moins 7 jours pour affirmer l'arrêt de grossesse, en l'absence d'apparition de l'activité cardiaque à l'échographie (grade C). 
Est-ce que le doppler a un intérêt dans le diagnostic d'une GIU arrêtée en cas de GIU d'évolutivité incertaine?

Aucune étude sur l'apport du doppler sur la prédiction d'évolution d'une GIU d'évolution incertaine n'a été identifiée. L'utilisation du doppler augmente l'énergie déposée dans les tissus par rapport à l'échographie en mode $\mathrm{B}$ conduisant à une augmentation thermique des tissus insonifiés (et aussi à un possible effet de cavitation). L'Index Thermique (IT) est utilisé pour mesurer l'augmentation de la température des tissus insonifiés. Il existe une augmentation négligeable de l'IT avec l'utilisation de l'échographie en mode B (en 2 D, 3D et 4D) du premier trimestre de grossesse. En mode doppler, il existe une augmentation importante de l'IT posant la question de l'innocuité du doppler pour les grossesses très précoces [41].

Aucune donnée n'est disponible pour définir la place du doppler pour le diagnostic d'évolutivité d'une GIU d'évolution incertaine au premier trimestre de grossesse.

Aucune donnée clinique n'existe sur l'innocuité du doppler au premier trimestre de grossesse. 
Est-ce que de l'hCG sérique a un intérêt dans le diagnostic d'une GIU arrêtée ou d'une GIU évolutive en cas de GIU d'évolutivité incertaine?

Un seul dosage de l'hCG sérique ne préjuge pas de l'évolutivité d'une grossesse, ni de sa localisation [42, 43] (NP2). La répétition du dosage des beta-hCG sérique n'a pas été étudiée dans la littérature pour définir l'évolution d'une grossesse intra-utérine d'évolution incertaine. 


\section{Quelle est la place du dosage sérique de l'hCG en cas de grossesse de localisation indéterminée ?}

En cas de grossesse de localisation indéterminée (définie par l'absence de grossesse visualisée à l'échographie chez une patiente avec un test de grossesse urinaire ou sanguin positif), l'enquête diagnostique doit orienter vers trois diagnostics: la grossesse ectopique, la grossesse intra-utérine évolutive ou la grossesse intra utérine arrêtée. Beaucoup de travaux ont étudiés la place des beta-hCG (et de leur cinétique) pour orienter vers ces trois diagnostics.

En cas de grossesse de localisation indéterminée, le dosage qualitatif urinaire des hCG a été étudié pour aider à l'interprétation de l'échographie endovaginale dans une étude rétrospective portant sur 254 patientes enceintes entre 5 et 8 semaines d'aménorrhée. La sensibilité et la spécificité pour l'exclusion d'une GIU évolutive étaient de $92 \%$ et $83 \%$ (NP3) respectivement [44]. Cette faible spécificité rend difficile l'utilisation seule du dosage urinaire qualitatif des hCG pour exclure une GIU évolutive.

En cas de grossesse de localisation indéterminée, le dosage quantitatif sérique des hCG a été étudié pour aider à l'interprétation de l'échographie endovaginale. Le seuil d'hCG sérique à partir duquel un sac gestationnel est visualisé par échographie endovaginale est variable : sur une étude rétrospective portant sur 651 grossesses dont 366 étaient évolutives chez des patientes symptomatiques (métrorragies ou douleurs), le seuil d'hCG sérique à partir duquel un sac gestationnel intra-utérin est vu dans 99\% des cas de GIU évolutive était de 3510 mUI/mL [45] (NP3). Le seuil minimal d'hCG à partir duquel un sac gestationnel intra-utérin a pu être observé était de $390 \mathrm{mUI} / \mathrm{mL}$ [45] (NP3). Ainsi, en cas de grossesse de localisation indéterminée à l'échographie endovaginale, un dosage sérique quantitatif de la beta-hCG > $3510 \mathrm{mUI} / \mathrm{mL}$ permet d'exclure une grossesse intra-utérine évolutive (NP3). Il s'agit soit d'une grossesse ectopique, soit d'une fausse couche complète. 
En cas de grossesse de localisation indéterminée à l'échographie endo-vaginale, on recommande d'utiliser un seuil du dosage des hCG sériques à au moins $3510 \mathrm{UI} / \mathrm{L}$ audessus duquel une grossesse intra-utérine évolutive peut être exclue (grade C).

Compte tenu des variations du dosage des hCG en fonction des kits de dosage des laboratoires (cf infra), chaque centre devrait évaluer ce seuil en fonction du laboratoire de biologie avec lequel il travaille.

En cas de grossesse de localisation indéterminée, la cinétique des beta-hCG (i.e. contrôle des beta-hCG à 48 heures d'intervalle) a été étudiée afin d'éliminer une grossesse intra-utérine évolutive. Dans une étude prospective de 49 grossesse non symptomatique, l'hCG sérique a doublé en moyenne tous les 1,4-1,6 jours depuis le début de détection jusqu'au $35^{\text {ième }}$ jour

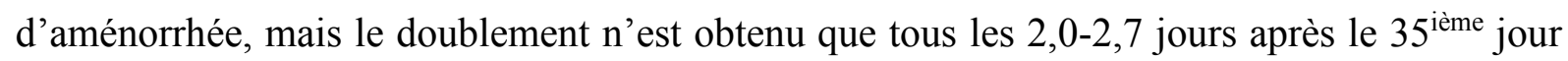
de grossesse [46] (NP2). Dans une étude prospective de 287 patientes symptomatiques (métrorragies ou douleurs) avec une échographie non informative à l'entrée et avec une grossesse prouvée évolutive dans le suivi, l'augmentation minimale des hCG à 2 jours d'intervalle était de 53\% [47] (NP2). Dans cette étude, la probabilité d'avoir une grossesse évolutive quand l'augmentation du taux de beta-hCG est inférieure à $53 \%$ à 48 heures d'intervalle était inférieure à 1\% [47] (NP2). Cependant, d'autres études ont trouvé des seuils d'augmentation plus restrictifs pour permettre d'exclure une grossesse évolutive. Dans une série rétrospective de 1249 patientes, Seeber et coll ont montré que pour avoir un risque inférieur à $0.1 \%$ d'avoir une grossesse évolutive, l'augmentation du taux de hCG doit être inférieure à $35 \%$ à 48 heures d'intervalle (NP3) [48]. Ce seuil d'augmentation de $35 \%$ à 48 heures a été évalué dans une autre série multicentrique prospective de 1000 patientes enceintes au premier trimestre et symptomatiques. Cette étude a montré que 20 patientes avaient encore une grossesse évolutive avec ce seuil à $35 \%$, soit un risque de $2 \%$ de grossesse évolutive malgré cette cinétique de beta-hCG «anormal », risque bien supérieur à $0,1 \%$ 
retrouvé par Seeber et coll. (NP2) [49]. Condous et coll ont développé un protocole sur une série rétrospective unicentrique de 500 patientes consécutives avec une grossesse de localisation indéterminée et l'ont validé sur une série prospective unicentrique de 503 patientes consécutives (NP2) [42]. Il a été montré que pour les patientes ayant un taux initial de hCG inférieur à $2000 \mathrm{UI} / \mathrm{L}$ et une augmentation inférieure ou égale à $15 \%$ au contrôle 48 heures après, une grossesse évolutive intra-utérine peut être formellement écartée (NP2) [42]. Entre 2000 et 3510 UI/L, les données publiées sur la cinétique des beta-hCG sont contradictoires et elles ne permettent pas de donner un seuil d'augmentation des beta-hCG au dessous duquel une grossesse intra-utérine évolutive pourrait être écartée.

En cas de hCG $<2000 \mathrm{UI} / \mathrm{L}$, on recommande un seuil d'augmentation des hCG au contrôle à 48 heures $\leq 15 \%$ pour écarter une grossesse intra-utérine évolutive (grade B).

En cas de grossesse de localisation indéterminée, la cinétique des beta-hCG (i.e. contrôle des beta-hCG à 48 heures d'intervalle) a été étudiée en cas de diagnostic de grossesse ectopique. Horne et coll ont montré dans une série prospective de 340 patientes symptomatiques au premier trimestre de grossesse que $10 \%$ des patientes avec une croissance normale des betahCG avaient une grossesse ectopique [50] (NP2). De même, Silva et coll ont montré que parmi 200 patientes avec le diagnostic de grossesse ectopique, $21 \%$ des patientes avaient une cinétique normale des beta-hCG (à savoir une augmentation d'au moins 53\% du taux de betahCG) [51] (NP2). A l'inverse, Horne et coll ont montré dans leur série que sur 214 patientes avec une chute des beta-hCG de plus de 10\%, 3\% des patientes avaient une grossesse ectopique [50]. De même, Silva et coll ont montré dans leur série de 200 patientes avec grossesse ectopique que $8 \%$ des patientes avaient une chute de leur beta-hCG imitant l'évolution d'une grossesse arrêtée [51]. En cas de grossesse de localisation indéterminée, 
une cinétique des beta-hCG croissante ou décroissante n'exclut pas une grossesse ectopique (NP2).

Dans une étude prospective de 106 dosages des beta-hCG sériques, il a été observé une variation du niveau des beta-hCG de $\pm 8,1 \%$ entre les différents kits de détection (NP2) [52]. Afin de comparer la cinétique des beta-hCG à 48 heures d'écart, les dosages doivent être effectués dans le même laboratoire avec le même kit de dosage pour éviter les variations dues aux différents kits de dosage (grade B). 
Quelle est la place de la progestéronémie dans la prise en charge des grossesses de localisation indéterminée à l’échographie endovaginale ?

Une méta-analyse portant sur 26 études de cohortes incluant 9436 patientes ( 7 études portant sur 2379 patientes présentant des douleurs pelviennes et/ou des métrorragies avec une grossesse de localisation inconnue à l'échographie et 19 études portant sur 7057 patientes présentant des douleurs pelviennes et/ou symptomatiques sans échographie) a été réalisée en 2012.

Sur 5 études portant sur 1998 patientes avec une grossesse de localisation indéterminée à l'échographie chez une femme avec des métrorragies et/ou des douleurs pelviennes, une progestéronémie inférieure de 3,2 à $6 \mathrm{ng} / \mathrm{mL}(5 \mathrm{ng} / \mathrm{mL}=16 \mathrm{nmol} / \mathrm{L})$ donnait une spécificité de diagnostic de grossesse non viable (i.e. exclure une grossesse intra utérine évolutive) de 98,4\% (95\%IC 90,9\% à 99,7\%) (NP1) [40]. Cependant, une progestéronémie inférieure au seuil retenu n'excluait pas une grossesse ectopique [40]. Une faible progestéronémie $(<3,2$ à $6 \mathrm{ng} / \mathrm{mL}$ ), chez une femme avec des métrorragies ou des douleurs présentant une grossesse de localisation indéterminée à l'échographie, exclut une grossesse intra-utérine évolutive à $98,4 \%(95 \% \mathrm{IC} 90,9 \%$ à 99,7\%) (NP1) [40].

Un dosage faible de la progestéronémie $(<3,2 \mathrm{ng} / \mathrm{mL})$ permet d'exclure une grossesse intra-utérine évolutive en cas de grossesse de localisation indéterminée (NP2).

En cas de dosage de la progestéronémie chez une patiente avec une grossesse de localisation indéterminée, un seuil inférieur à $3,2 \mathrm{ng} / \mathrm{mL}$ permet d'exclure une grossesse intra-utérine évolutive (Grade B). 
Quels sont les facteurs pronostiques d'évolutivité chez les patientes enceintes symptomatiques avec un diagnostic de grossesse d'évolutivité incertaine?

Des éléments cliniques et échographiques sont pronostiques pour l'évolution de la grossesse. Ces éléments pronostiques ont été décrits dans une étude de cohorte prospective unicentrique portant sur 1435 patientes se présentant dans des unités de type «early pregnancy clinic » (NP2) [53] et dans une étude rétrospective unicentrique non consécutive de cohorte portant sur 480 patientes avec un diagnostic de grossesse d'évolution incertaine (analyse uni et multivariée) [54] (NP4). Un âge maternel plus avancé (>35 ans), l'antécédent de grossesse arrêtée au premier trimestre, un âge gestationnel plus avancé, des métrorragies (d'autant plus s'il y a des caillots), une taille de sac gestationnel plus grand ou une vésicule vitelline $>5 \mathrm{~mm}$ à l'échographie sont des critères de mauvais pronostic. D'autres éléments pronostiques ont pu être identifiés: une taille embryonnaire inférieure à la taille attendue pour le terme gestationnel est significativement associée à une grossesse arrêtée [21, 22, 55-57] (NP2). L'hématome sous chorial n'est pas un facteur pronostique indépendant de grossesse arrêtée ultérieure si les métrorragies sont prises en comptes [58] (NP4).

Des scores et des modèles de prédiction d'une grossesse arrêtée ou d'une grossesse évolutive dans le suivi des patientes [22-24, 53, 54], dont certains avec des validations externes sur 575 et 1435 patientes [23, 53], ont été développés combinant les éléments cliniques et échographiques. Ils permettent de donner un pronostic individuel de grossesse arrêtée ou évolutive à une patiente mais ne peuvent affirmer à eux seuls une grossesse évolutive ou une grossesse arrêtée dans le suivi ultérieur, ce qui en limite l'intérêt. Ces scores ne modifient pas la prise en charge des patientes avec un diagnostic de grossesse d'évolutivité incertaine. 
Quels sont les facteurs pronostiques d'évolutivité chez les patientes enceintes présentant des métrorragies ou des douleurs au premier trimestre de grossesse avec un diagnostic de grossesse évolutive (activité cardiaque vue)?

Le taux de grossesse arrêtée après la visualisation initiale d'une activité cardiaque au premier trimestre est de 2 à 16\% (NP2) [59-62]. Des scores ou modèles de prédiction d'une évolution favorable ou de la survenue d'une grossesse arrêtée ont été développés chez des patientes avec un diagnostic échographique de grossesse évolutive (activité cardiaque vue). Ces scores ou modèles ne permettent pas d'éviter un second contrôle échographique pour déterminer le devenir ultérieur de la grossesse (grossesse évolutive ou grossesse arrêtée). 
Dans quel délai faut-il réaliser une seconde échographie chez une patiente présentant des métrorragies ou des douleurs avec une grossesse évolutive échographique?

La littérature ne permet pas de déterminer de délai optimal pour effectuer une seconde échographie et permettre de donner le diagnostic de grossesse arrêtée ou évolutive ultérieure chez une patiente présentant une menace de fausse couche (patientes enceinte au premier trimestre et présentant des métrorragies). Il n'existe pas d'étude déterminant le délai à respecter pour refaire une seconde échographie d'évolutivité de la grossesse afin de pouvoir affirmer le devenir de la grossesse, en cas de patientes présentant des métrorragies et/ou des douleurs et une grossesse intra-utérine évolutive à l'échographie. 
Quels sont les éléments nécessaires à mentionner dans un compte rendu d'échographie pelvienne réalisée pour une GIU dans le premier trimestre chez une patiente présentant des douleurs ou des métrorragies?

Actuellement, aucun compte rendu standardisé n'existe pour la description d'une GIU au premier trimestre de grossesse avant $10 \mathrm{SA}$ chez une femme présentant des douleurs ou des métrorragies. Compte tenu des différentes informations nécessaires pour le diagnostic de grossesse arrêtée, le compte rendu de l'échographie doit indiquer :

- Des données démographiques et clinico-biologiques :

- Age de la patiente

○ La date des dernières règles ou potentielle de conception en cas d'assistance médicale à la procréation

○ L'âge gestationnel théorique en semaine d'aménorrhée

○ La présence de symptômes (métrorragies et/ou douleurs)

- La date et le taux des beta-hCG si dosés

- Les conditions d'examen :

○ Voie endo-vaginale et/ou sus-pubienne

- Les informations sur la grossesse :

- La taille moyenne du sac gestationnel intra-utérin (moyenne des 3 mesures orthogonales)

○ La présence ou l'absence de Vésicule Vitelline

- La présence ou l'absence d'un embryon.

- Si l'embryon est présent, la mesure de la longueur cranio-caudale

○ Si l'embryon est présent, l'absence ou la présence d'une activité cardiaque

- Les informations suivantes :

- Aspect des ovaires 
○ Présence ou non d'un épanchement péritonéal (au niveau du cul de sac de Douglas et/ou dans la loge de Morrison)

○ Présence ou non d'une masse latéro-utérine

La conclusion de l'échographie chez une femme enceinte avant 10 SA et présentant des douleurs et/ou des métrorragies devrait indiquer le statut de la grossesse selon les termes suivant (tableau 2) :

○ Grossesse intra-utérine évolutive

○ Grossesse intra-utérine d'évolutivité incertaine

- Grossesse intra-utérine arrêtée

- Grossesse de localisation indéterminée

○ Grossesse ectopique 


\section{Quels moyens de prévention en cas de grossesse intra-utérine évolutive avec des}

métrorragies au premier trimestre de grossesse (menace de fausse couche) (hors cadre du syndrome des fausses couches à répétition ou d'un contexte d'assistance médicale à la procréation)?

L'impact du repos allongé sur le devenir de la grossesse a été évalué au travers d'une métaanalyse publiée en 2005 qui a identifié seulement deux études portant sur 84 patientes [63]. Il n’a pas été montré de bénéfice significatif du repos allongé strict (à domicile ou en hospitalisation) sur le devenir de la grossesse dans ces 2 études de faible effectif (NP3). Compte tenu du faible effectif, les auteurs de la méta-analyse concluent à l'absence de preuve sur le bénéfice ou non du repos allongé sur la survenue d'une grossesse arrêtée [63].

Le rôle de la supplémentation vitaminique (quelle que soit sa nature : vitamine A (5000 UI à 23300 UI, Acide folique (0,3 à 10mg), vitamine C (400mg à $1000 \mathrm{mg})$, soit seule ou en association entre elles et/ou avec une supplémentation martiale et/ou avec de la vitamine E) sur la prévention d'une grossesse arrêtée en cas de menace de fausse couche (métrorragies et/ou douleurs au premier trimestre) a été évalué dans une méta-analyse publiée en 2011 qui a identifié 28 études portant sur 96,674 femmes [64]. Cette méta-analyse a regroupé les données de 3 principaux essais portant sur 62,669 patientes. Aucune différence significative n'a pu être mise en évidence sur la survenue d'une grossesse arrêtée avec ou sans supplémentation vitaminique [64] (NP1).

L'utilisation de la progestérone dans la prévention de la grossesse arrêtée a été évaluée par une méta-analyse publiée en 2013, qui a identifié 14 essais cliniques randomisés portant sur 2158 patientes [65]. Ces 14 essais concernaient des patientes asymptomatiques avec des antécédents de fausses couches (7 essais), des patientes enceintes avec des métrorragies et/ou des douleurs avec ou sans antécédent de fausses couches (5 essais), des patientes au décours d'une amniocentèse (1 essai) et des patientes dans un contexte d'assistance médicale à la 
procréation (1 essai). Il n'a pas été montré de différence significative sur la survenue de grossesse arrêtée entre le groupe prenant de la progestérone et le groupe contrôle (NP1). La voie d'administration de la progestérone (orale, intra-musculaire ou vaginale) n'a pas montré de différence significative sur la prévention de l'arrêt de grossesse [65]. Les études concernant seulement les patientes avec des menaces de fausses couches (métrorragies ou douleurs au premier trimestre de grossesse) n'ont pas mis en évidence de bénéfice à une supplémentation en progestérone sur la prévention d'une fausse couche [66-68]. Quatre essais de mauvaise qualité ont évalué l'utilisation de la progestérone dans le cadre des fausses couches à répétition (c'est à dire $\geq 3$ fausses couches) et ont montré un certain bénéfice à la prise de progestérone dans ce contexte. Le contexte des fausses couches à répétition est traité dans un autre chapitre de ces recommandations pour la pratique clinique (chapitre 7, [69]).

L'utilisation de l'human chorionic gonadotrophin (hCG) pour prévenir la survenue d'une grossesse arrêtée en cas de menace de fausse couche (métrorragies et/ou douleurs au premier trimestre de grossesse) a été évaluée dans une méta-analyse publiée en 2013 et qui a identifié 5 études portant sur 596 patientes, dont deux étude de mauvaise qualité. Les auteurs concluent a des résultats équivoques empêchant de conclure au bénéfice ou l'absence de bénéfice du traitement par hCG pour prévenir une fausse couche [70].

L'utilisation des herbes chinoises pour prévenir la survenue d'une grossesse arrêtée an cas de menace de fausse couche a été évaluée par une méta-analyse publiée en 2012 qui a identifié 44 essais portant sur 5100 patientes [71]. Il n'existe pas d'essai randomisant les herbes chinoises versus aucun traitement. Les essais randomisant les herbes chinoises versus une médecine occidentale (comme l'utilisation de l'hCG, la progestérone, une supplémentation vitaminique, une immunothérapie, des beta2-mimétiques) n'ont pas montré une supériorité de l'un ou l'autre des traitements. 
Aucune supplémentation vitaminique n'a fait la preuve de son efficacité pour diminuer le risque de survenue d'une grossesse arrêtée en cas de grossesse intra-utérine chez une femme présentant des métrorragies ou des douleurs (NP1).

Un traitement par progestérone ne diminue pas le risque de survenue d'une grossesse arrêtée en cas de grossesse intra-utérine chez une femme présentant des métrorragies ou des douleurs (en dehors du contexte des fausses couches à répétition) (NP1).

Les données sont insuffisantes pour conclure au bénéfice ou l'absence de bénéfice du traitement par hCG sur la survenue d'une grossesse arrêtée en cas de grossesse intrautérine chez une femme présentant des métrorragies ou des douleurs.

Il n'existe pas de preuve sur le bénéfice ou l'absence de bénéfice des herbes chinoises sur le risque de survenue d'une grossesse arrêtée en cas de grossesse intra-utérine chez une femme présentant des métrorragies ou des douleurs.

Il n'existe pas de preuve sur le bénéfice du repos allongé sur le risque de survenue d'une grossesse arrêtée en cas de grossesse intra-utérine chez une femme présentant des métrorragies ou des douleurs.

Il n'est recommandé ni supplémentation en vitamine, ni traitement par progestérone ou hCG, ni repos allongé en cas de grossesse intra-utérine chez une femme présentant des métrorragies ou des douleurs, qu'elles aient ou non un antécédent de fausse couche (grade B). 
En cas de grossesse intra-utérine arrêtée au premier trimestre de grossesse, quelle est la place de l'examen histologique de produit de conception et quelle est la place du caryotype du produit de conception?

En cas d'aspiration chirurgicale d'une grossesse arrêtée, la rentabilité diagnostique de l'analyse histologique du produit de conception n'est pas connue, que ce soit pour confirmer le diagnostic de grossesse intra-utérine arrêtée, éliminer une grossesse extra-utérine ou éliminer une grossesse môlaire. Il n'existe pas non plus d'analyse médico-économique sur l'analyse histologique du produit de conception.

En cas de grossesse arrêtée, le taux d'anomalie chromosomique pour une première fausse couche oscille entre 45 et $68 \%[72,73]$ (NP2). Le risque de contamination du caryotype du produit de conception par des cellules maternelles faussant ainsi le diagnostic caryotypique est important : plus de la moitié des caryotypes $46 \mathrm{XX}$ (et considérés comme normaux) sont en fait dus à une contamination par des cellules maternelles (NP2) [73]. Aucune étude n'est disponible pour évaluer l'intérêt du caryotype sur le produit de conception en cas de fausse couche « sporadique ».

Il n'existe pas de donnée dans la littérature pour préconiser une analyse histologique du produit de FC, en dehors des suspicions de tumeurs trophoblastiques gestationnelles. Il n'est pas recommandé de réaliser d'étude caryotypique du produit de fausse couche (Grade C). 
Quel est le délai à respecter après une grossesse arrêtée avant d'entamer une nouvelle grossesse ?

Les règles réapparaissent entre 1 ou 2 mois après une fausse couche. Une étude rétrospective portant sur 30,937 patientes ayant eu une fausse couche spontanée du premier trimestre et ensuite une nouvelle grossesse a montré que les patientes ayant une nouvelle grossesse dans les 6 mois après une fausse couche, ont moins souvent de nouvelle fausse couche comparées aux patientes ayant une nouvelle grossesse dans les 6 à 12 mois [74] (NP3). La survenue d'une nouvelle grossesse dans les 6 mois après une fausse couche spontanée diminue le risque de nouvelle fausse couche de $34 \%$ (Intervalle de confiance (IC) à $95 \%$ de $23 \%$ à $43 \%$ ) de manière indépendante. Le taux de complications dans la grossesse suivante est également diminué. En effet, en cas de nouvelle grossesse dans les 6 mois, le risque de césarienne est diminué de $10 \%(\mathrm{OR}=0,90$, avec un IC à $95 \%$ entre 0,83 et 0,98$)$, de même que le risque d'accouchement prématuré (OR à 0,89 avec un IC entre 0,81 to 0,98 ) et le risque de retard de croissance intra-utérin $(0,84$ avec un IC entre 0,81 et 0,98$)$. Le risque de déclenchement du travail est par contre augmenté avec un OR à 1,08 (IC entre 1,02 et 1,23) pour les grossesses survenant dans les 6 mois après une fausse couche. Ces données ont été confirmées par une étude prospective portant sur 4619 patientes en analyse multivariée [75] (NP1).

La survenue d'une nouvelle grossesse dans les 6 mois après une fausse couche précoce est associée à un risque moindre de survenue d'une nouvelle fausse couche et de complications pour cette nouvelle grossesse (NP1).

En cas de fausse couche précoce et de souhait de la patiente d'une nouvelle grossesse, il est recommandé de ne pas différer la survenue de cette nouvelle grossesse (grade A). 
Quelle est la place de la pilule oestro-progestative après une grossesse arrêtée ?

Aucune donnée n'a été identifiée sur l'intérêt ou l'absence d'intérêt de la prescription d'une pilule oestro-progestative au décours d'une grossesse arrêtée. 


\section{Conclusion}

Le diagnostic de grossesse arrêtée au premier trimestre repose sur la mesure du sac gestationnel (mesurée sur 3 plans de coupe orthogonaux en échographie endovaginale) et la mesure de la longueur cranio-caudale de l'embryon à l'échographie endovaginale (Grade B). On recommande un seuil $\geq 25 \mathrm{~mm}$ pour la mesure moyenne échographique (voie endovaginale) du sac gestationnel intra-utérin sans embryon pour affirmer l'arrêt d'une grossesse intra-utérine (Grade B). On recommande un seuil $\geq 7 \mathrm{~mm}$ pour la mesure de la longueur cranio-caudale d'un embryon mesurée dans le plan sagittal par échographie endovaginale pour affirmer l'arrêt d'une grossesse intra-utérine (Grade B). La grossesse intrautérine d'évolutivité incertaine est définie à l'échographie endovaginale par un sac gestationnel intra-utérin sans critère échographique de grossesse arrêtée ou de grossesse évolutive (activité cardiaque visualisée). En cas de grossesse intra-utérine d'évolutivité incertaine, on recommande un contrôle échographique réalisé à plus de 7 à 14 jours en fonction de la présence ou non d'une vésicule vitelline ou d'un embryon à l'échographie initiale permettra d'exclure une grossesse évolutive intra-utérine (Grade C). Il n'existe pas de moyen connu de prévention efficace d'une fausse couche en cas de métrorragies et/ou de douleur chez une femme enceinte au premier trimestre. Si une patiente souhaite une nouvelle grossesse après une fausse couche précoce, on recommande la survenue de cette grossesse dans les 6 premiers mois après la fausse couche pour diminuer le risque de nouvelle fausse couche par rapport à la survenue d'une nouvelle grossesse au-delà de 6 mois (grade A). 


\section{Références}

1. Wieringa-de Waard, M., et al., Threatened miscarriage in general practice: diagnostic value of history taking and physical examination. Br J Gen Pract, 2002. 52(483): p. 825-9.

2. Doubilet, P.M., et al., Diagnostic criteria for nonviable pregnancy early in the first trimester. N Engl J Med, 2013. 369(15): p. 1443-51.

3. Timor-Tritsch, I.E., D. Farine, and M.G. Rosen, $A$ close look at early embryonic development with the high-frequency transvaginal transducer. Am J Obstet Gynecol, 1988. 159(3): p. 676-81.

4. Bree, R.L., et al., Transvaginal sonography in the evaluation of normal early pregnancy: correlation with HCG level. AJR Am J Roentgenol, 1989. 153(1): p. 759.

5. Warren, W.B., et al., Dating the early pregnancy by sequential appearance of embryonic structures. Am J Obstet Gynecol, 1989. 161(3): p. 747-53.

6. Coulam, C.B., S. Britten, and D.M. Soenksen, Early (34-56 days from last menstrual period) ultrasonographic measurements in normal pregnancies. Hum Reprod, 1996. 11(8): p. 1771-4.

7. Jauniaux, E., et al., Development of the secondary human yolk sac: correlation of sonographic and anatomical features. Hum Reprod, 1991. 6(8): p. 1160-6.

8. Britten, S., et al., Very early (24-56 days from last menstrual period) embryonic heart rate in normal pregnancies. Hum Reprod, 1994. 9(12): p. 2424-6.

9. Robinson, H.P., Sonar measurement of fetal crown-rump length as means of assessing maturity in first trimester of pregnancy. Br Med J, 1973. 4(5883): p. 2831.

10. Robinson, H.P. and J.E. Fleming, A critical evaluation of sonar "crown-rump length" measurements. Br J Obstet Gynaecol, 1975. 82(9): p. 702-10.

11. Hadlock, F.P., et al., Fetal crown-rump length: reevaluation of relation to menstrual age (5-18 weeks) with high-resolution real-time US. Radiology, 1992. 182(2): p. 501-5.

12. Daya, S., Accuracy of gestational age estimation by means of fetal crown-rump length measurement. Am J Obstet Gynecol, 1993. 168(3 Pt 1): p. 903-8.

13. Wisser, J., P. Dirschedl, and S. Krone, Estimation of gestational age by transvaginal sonographic measurement of greatest embryonic length in dated human embryos. Ultrasound Obstet Gynecol, 1994. 4(6): p. 457-62.

14. Kustermann, A., et al., Transvaginal sonography for fetal measurement in early pregnancy. Br J Obstet Gynaecol, 1992. 99(1): p. 38-42.

15. Bottomley, C. and T. Bourne, Dating and growth in the first trimester. Best Pract Res Clin Obstet Gynaecol, 2009. 23(4): p. 439-52.

16. Pexsters, A., et al., New crown-rump length curve based on over 3500 pregnancies. Ultrasound Obstet Gynecol, 2010. 35(6): p. 650-5.

17. Hellman, L.M., et al., Growth and development of the human fetus prior to the twentieth week of gestation. Am J Obstet Gynecol, 1969. 103(6): p. 789-800.

18. Goldstein, I., et al., Evaluation of normal gestational sac growth: appearance of embryonic heartbeat and embryo body movements using the transvaginal technique. Obstet Gynecol, 1991. 77(6): p. 885-8.

19. Oh, J.S., G. Wright, and C.B. Coulam, Gestational sac diameter in very early pregnancy as a predictor of fetal outcome. Ultrasound Obstet Gynecol, 2002. $20(3)$ : p. 267-9. 
20. Abdallah, Y., et al., Limitations of current definitions of miscarriage using mean gestational sac diameter and crown-rump length measurements: a multicenter observational study. Ultrasound Obstet Gynecol, 2011. 38(5): p. 497-502.

21. Bottomley, C., et al., Functional linear discriminant analysis: a new longitudinal approach to the assessment of embryonic growth. Hum Reprod, 2009. 24(2): p. 278-83.

22. Choong, S., et al., Ultrasound prediction of risk of spontaneous miscarriage in live embryos from assisted conceptions. Ultrasound Obstet Gynecol, 2003. 22(6): p. 571-7.

23. Guha, S., et al., External validation of models and simple scoring systems to predict miscarriage in intrauterine pregnancies of uncertain viability. Hum Reprod, 2013. 28(11): p. 2905-11.

24. Oates, J., et al., A prediction model for viability at the end of the first trimester after a single early pregnancy evaluation. Aust N Z J Obstet Gynaecol, 2013. 53(1): p. 51-7.

25. Pexsters, A., et al., Clinical implications of intra- and interobserver reproducibility of transvaginal sonographic measurement of gestational sac and crown-rump length at 6-9 weeks' gestation. Ultrasound Obstet Gynecol, 2011. 38(5): p. 510-5.

26. Verburg, B.O., et al., Intra- and interobserver reproducibility study of early fetal growth parameters. Prenat Diagn, 2008. 28(4): p. 323-31.

27. Ferrazzi, E., et al., Miscarriage diagnosis and gestational age estimation in the early first trimester of pregnancy: transabdominal versus transvaginal sonography. Ultrasound Obstet Gynecol, 1993. 3(1): p. 36-41.

28. Jain, K.A., U.M. Hamper, and R.C. Sanders, Comparison of transvaginal and transabdominal sonography in the detection of early pregnancy and its complications. AJR Am J Roentgenol, 1988. 151(6): p. 1139-43.

29. Pennell, R.G., et al., Prospective comparison of vaginal and abdominal sonography in normal early pregnancy. J Ultrasound Med, 1991. 10(2): p. 63-7.

30. Cacciatore, B., U.H. Stenman, and P. Ylostalo, Comparison of abdominal and vaginal sonography in suspected ectopic pregnancy. Obstet Gynecol, 1989. 73(5 Pt 1): p. 770-4.

31. Bradley, W.G., C.E. Fiske, and R.A. Filly, The double sac sign of early intrauterine pregnancy: use in exclusion of ectopic pregnancy. Radiology, 1982. 143(1): p. 2236.

32. Yeh, H.C., et al., Intradecidual sign: a US criterion of early intrauterine pregnancy. Radiology, 1986. 161(2): p. 463-7.

33. Laing, F.C., et al., Intradecidual sign: is it effective in diagnosis of an early intrauterine pregnancy? Radiology, 1997. 204(3): p. 655-60.

34. Chiang, G., et al., The intradecidual sign: is it reliable for diagnosis of early intrauterine pregnancy? AJR Am J Roentgenol, 2004. 183(3): p. 725-31.

35. Doubilet, P.M. and C.B. Benson, Double sac sign and intradecidual sign in early pregnancy: interobserver reliability and frequency of occurrence. J Ultrasound Med, 2013. 32(7): p. 1207-14.

36. Mueller, C.E., Intrauterine pseudogestational sac in ectopic pregnancy. J Clin Ultrasound, 1979. 7(2): p. 133-6.

37. Crochet, J.R., L.A. Bastian, and M.V. Chireau, Does this woman have an ectopic pregnancy?: the rational clinical examination systematic review. JAMA, 2013. 309(16): p. 1722-9. 
38. Fernandez, H. and A. Gervaise, Ectopic pregnancies after infertility treatment: modern diagnosis and therapeutic strategy. Hum Reprod Update, 2004. 10(6): p. 503-13.

39. Jeve, Y., et al., Accuracy of first-trimester ultrasound in the diagnosis of early embryonic demise: a systematic review. Ultrasound Obstet Gynecol, 2011. 38(5): p. 489-96.

40. Verhaegen, J., et al., Accuracy of single progesterone test to predict early pregnancy outcome in women with pain or bleeding: meta-analysis of cohort studies. BMJ, 2012. 345: p. e6077.

41. Sheiner, E. and J.S. Abramowicz, A symposium on obstetrical ultrasound: is all this safe for the fetus? Clin Obstet Gynecol, 2012. 55(1): p. 188-98.

42. Condous, G., et al., Prediction of ectopic pregnancy in women with a pregnancy of unknown location. Ultrasound Obstet Gynecol, 2007. 29(6): p. 680-7.

43. Kirk, E., et al., Rationalizing the follow-up of pregnancies of unknown location. Hum Reprod, 2007. 22(6): p. 1744-50.

44. Mazouz, S., J.K. Lee, and H. Fernandez, Evaluation of a urinary test as a diagnostic tool of a nonprogressive pregnancy. Fertil Steril, 2011. 95(2): p. 783-6.

45. Connolly, A., et al., Reevaluation of discriminatory and threshold levels for serum beta-hCG in early pregnancy. Obstet Gynecol, 2013. 121(1): p. 65-70.

46. Pittaway, D.E., R.L. Reish, and A.C. Wentz, Doubling times of human chorionic gonadotropin increase in early viable intrauterine pregnancies. Am J Obstet Gynecol, 1985. 152(3): p. 299-302.

47. Barnhart, K.T., et al., Symptomatic patients with an early viable intrauterine pregnancy: HCG curves redefined. Obstet Gynecol, 2004. 104(1): p. 50-5.

48. Seeber, B.E., et al., Application of redefined human chorionic gonadotropin curves for the diagnosis of women at risk for ectopic pregnancy. Fertil Steril, 2006. 86(2): p. 454-9.

49. Morse, C.B., et al., Performance of human chorionic gonadotropin curves in women at risk for ectopic pregnancy: exceptions to the rules. Fertil Steril, 2012. 97(1): p. 101-6 e2.

50. Horne, A.W., R. McBride, and F.C. Denison, Normally rising hCG does not predict live birth in women presenting with pain and bleeding in early pregnancy. Eur J Obstet Gynecol Reprod Biol, 2011. 156(1): p. 120-1.

51. Silva, C., et al., Human chorionic gonadotropin profile for women with ectopic pregnancy. Obstet Gynecol, 2006. 107(3): p. 605-10.

52. Massart, C., et al., [Application of a rapid serum beta-hCG assay in the early diagnosis of the ectopic pregnancies (author's transl)]. J Gynecol Obstet Biol Reprod (Paris), 1981. 10(7): p. 675-81.

53. Bottomley, C., et al., Accurate prediction of pregnancy viability by means of a simple scoring system. Hum Reprod, 2013. 28(1): p. 68-76.

54. Lautmann, K., et al., Clinical use of a model to predict the viability of early intrauterine pregnancies when no embryo is visible on ultrasound. Hum Reprod, 2011. 26(11): p. 2957-63.

55. Mantoni, M. and J.F. Pedersen, Fetal growth delay in threatened abortion: an ultrasound study. Br J Obstet Gynaecol, 1982. 89(7): p. 525-7.

56. Reljic, M., The significance of crown-rump length measurement for predicting adverse pregnancy outcome of threatened abortion. Ultrasound Obstet Gynecol, 2001. 17(6): p. 510-2. 
57. Mukri, F., et al., Evidence of early first-trimester growth restriction in pregnancies that subsequently end in miscarriage. BJOG, 2008. 115(10): p. 1273-8.

58. Johns, J., J. Hyett, and E. Jauniaux, Obstetric outcome after threatened miscarriage with and without a hematoma on ultrasound. Obstet Gynecol, 2003. 102(3): p. 483-7.

59. Cashner, K.A., C.R. Christopher, and G.A. Dysert, Spontaneous fetal loss after demonstration of a live fetus in the first trimester. Obstet Gynecol, 1987. 70(6): p. 827-30.

60. Siddiqi, T.A., et al., Rate of spontaneous abortion after first trimester sonographic demonstration of fetal cardiac activity. Am J Perinatol, 1988. 5(1): p. 1-4.

61. Hill, L.M., et al., Fetal loss rate after ultrasonically documented cardiac activity between 6 and 14 weeks, menstrual age. J Clin Ultrasound, 1991. 19(4): p. 221-3.

62. Makrydimas, G., et al., Fetal loss following ultrasound diagnosis of a live fetus at 610 weeks of gestation. Ultrasound Obstet Gynecol, 2003. 22(4): p. 368-72.

63. Aleman, A., et al., Bed rest during pregnancy for preventing miscarriage. Cochrane Database Syst Rev, 2005(2): p. CD003576.

64. Rumbold, A., et al., Vitamin supplementation for preventing miscarriage. Cochrane Database Syst Rev, 2011(1): p. CD004073.

65. Haas, D.M. and P.S. Ramsey, Progestogen for preventing miscarriage. Cochrane Database Syst Rev, 2013. 10: p. CD003511.

66. Gerhard, I., et al., Double-blind controlled trial of progesterone substitution in threatened abortion. Biol Res Pregnancy Perinatol, 1987. 8(1 1ST Half): p. 26-34.

67. Reijnders, F.J., et al., Endocrine effects of 17 alpha-hydroxyprogesterone caproate during early pregnancy: a double-blind clinical trial. Br J Obstet Gynaecol, 1988. 95(5): p. 462-8.

68. Tognoni, G., et al., Progestagens in threatened abortion. Lancet, 1980. 2(8206): p. 1242-3.

69. Gallot, V., et al., Fausses couches précoces "à répétition" : bilan et prise en charge. 2014.

70. Morley, L.C., N. Simpson, and T. Tang, Human chorionic gonadotrophin (hCG) for preventing miscarriage. Cochrane Database Syst Rev, 2013. 1: p. CD008611.

71. Li, L., et al., Chinese herbal medicines for threatened miscarriage. Cochrane Database Syst Rev, 2012. 5: p. CD008510.

72. van den Berg, M.M., et al., Genetics of early miscarriage. Biochim Biophys Acta, 2012. 1822(12): p. 1951-9.

73. Lathi, R.B., et al., Reliability of 46,XX results on miscarriage specimens: a review of 1,222 first-trimester miscarriage specimens. Fertil Steril, 2014. 101(1): p. 178-82.

74. Love, E.R., et al., Effect of interpregnancy interval on outcomes of pregnancy after miscarriage: retrospective analysis of hospital episode statistics in Scotland. BMJ, 2010. 341: p. c3967.

75. El Behery, M.M., et al., Reproductive performance in the next pregnancy for nulliparous women with history of first trimester spontaneous abortion. Arch Gynecol Obstet, 2013. 288(4): p. 939-44. 
Figure 1 : Premières structures identifiables de grossesse à l'échographie endovaginale

Figure 1: First transvaginal ultrasonographic signs of pregnancy 1a)

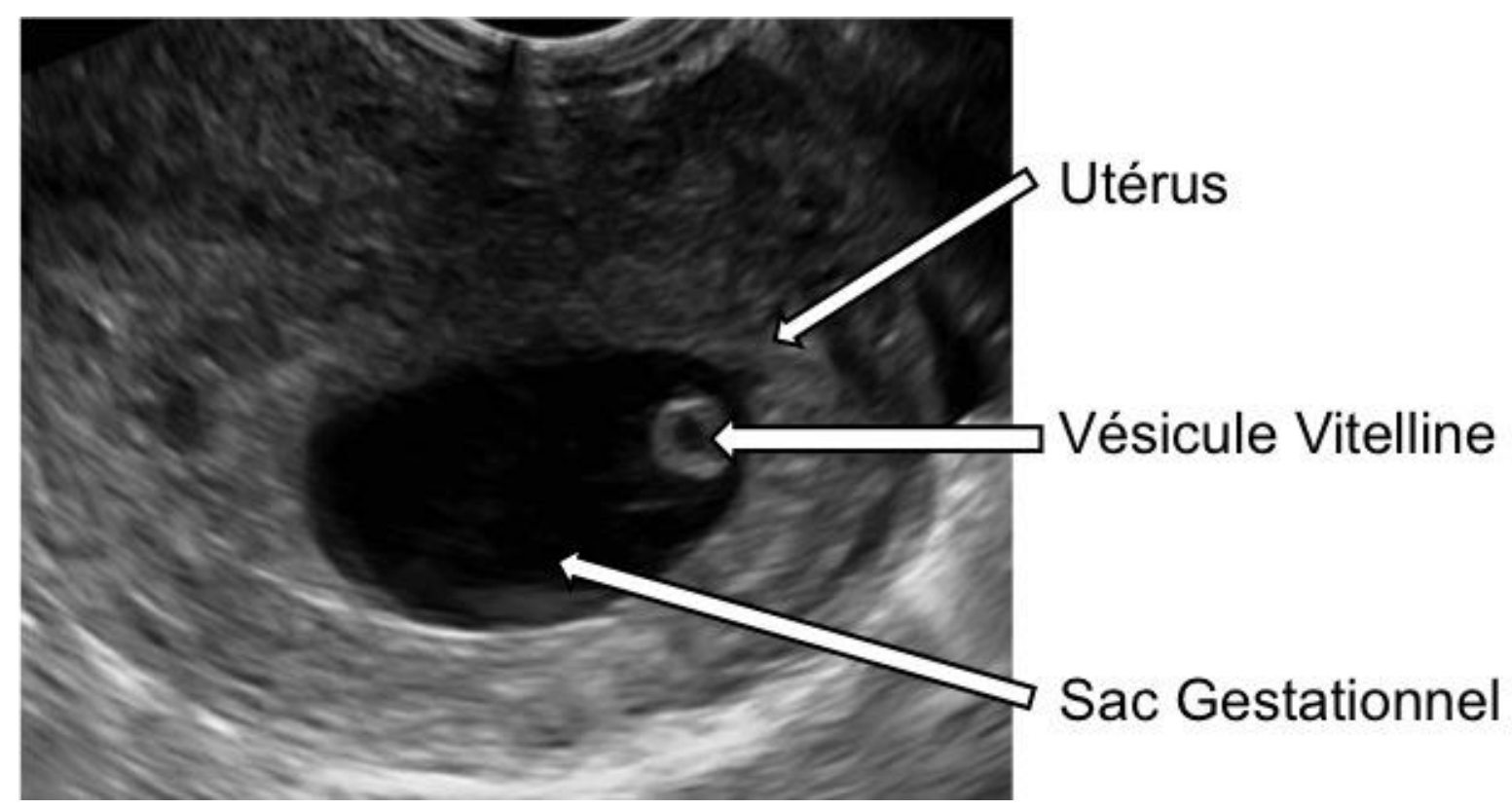

1b)

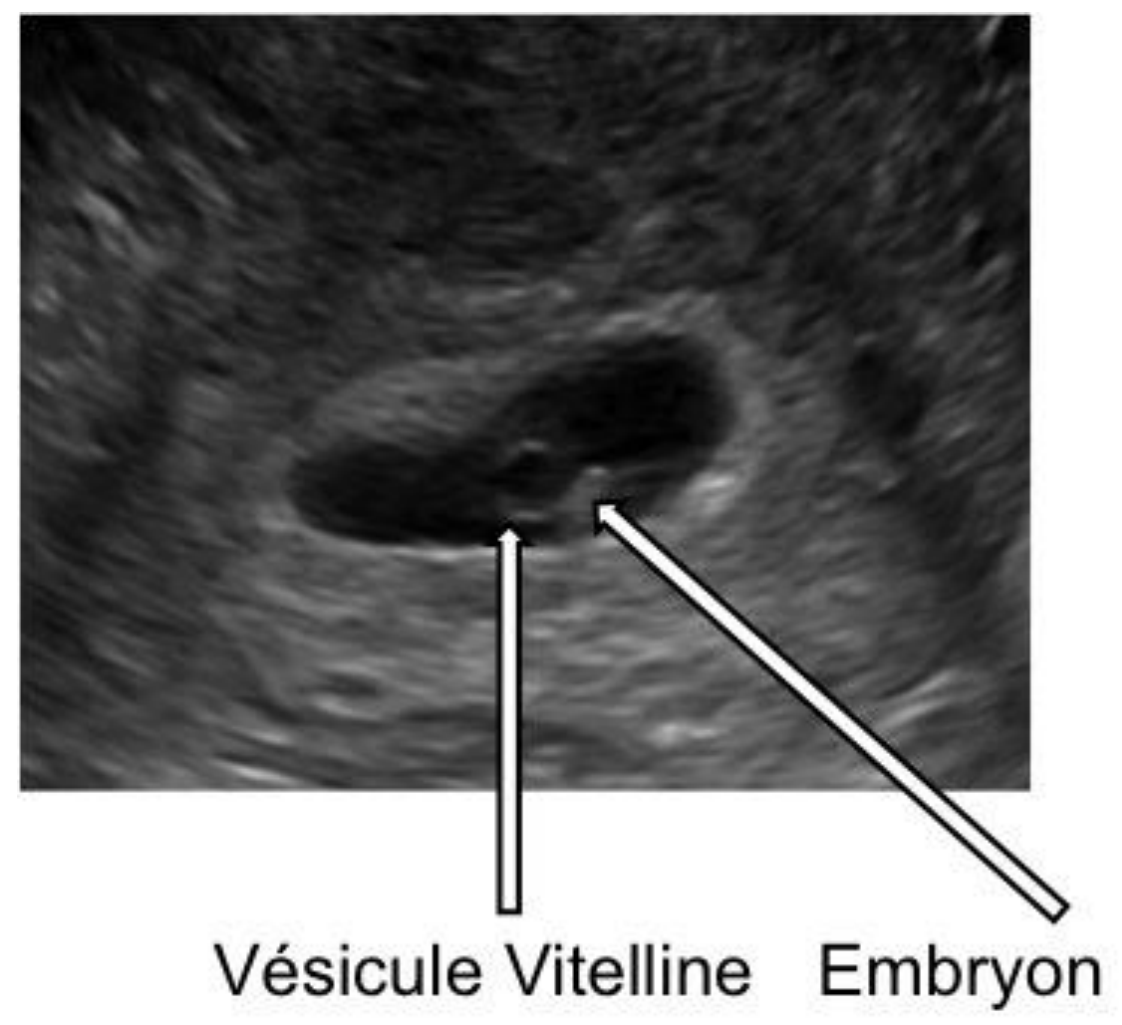


Légende figure 1 : échographie endovaginale au premier trimestre de grossesse (6 Semaines d'aménorrhée). a)Le sac gestationnel apparait sous forme d'une image anéchogène arrondie entouré par un anneau échogène. La vésicule vitelline apparait sous la forme d'un anneau anéchogène excentré dans le sac gestationnel ; b) Le pole embryonnaire apparait sou sforme d'une structure linéaire échogène à la périphérie de la vésicule vitelline. 
Figure 2: Position des calipers pour la mesure du sac gestationnel en échographie Figure 2: Place of calipers for gestational sac measurement with ultrasonography
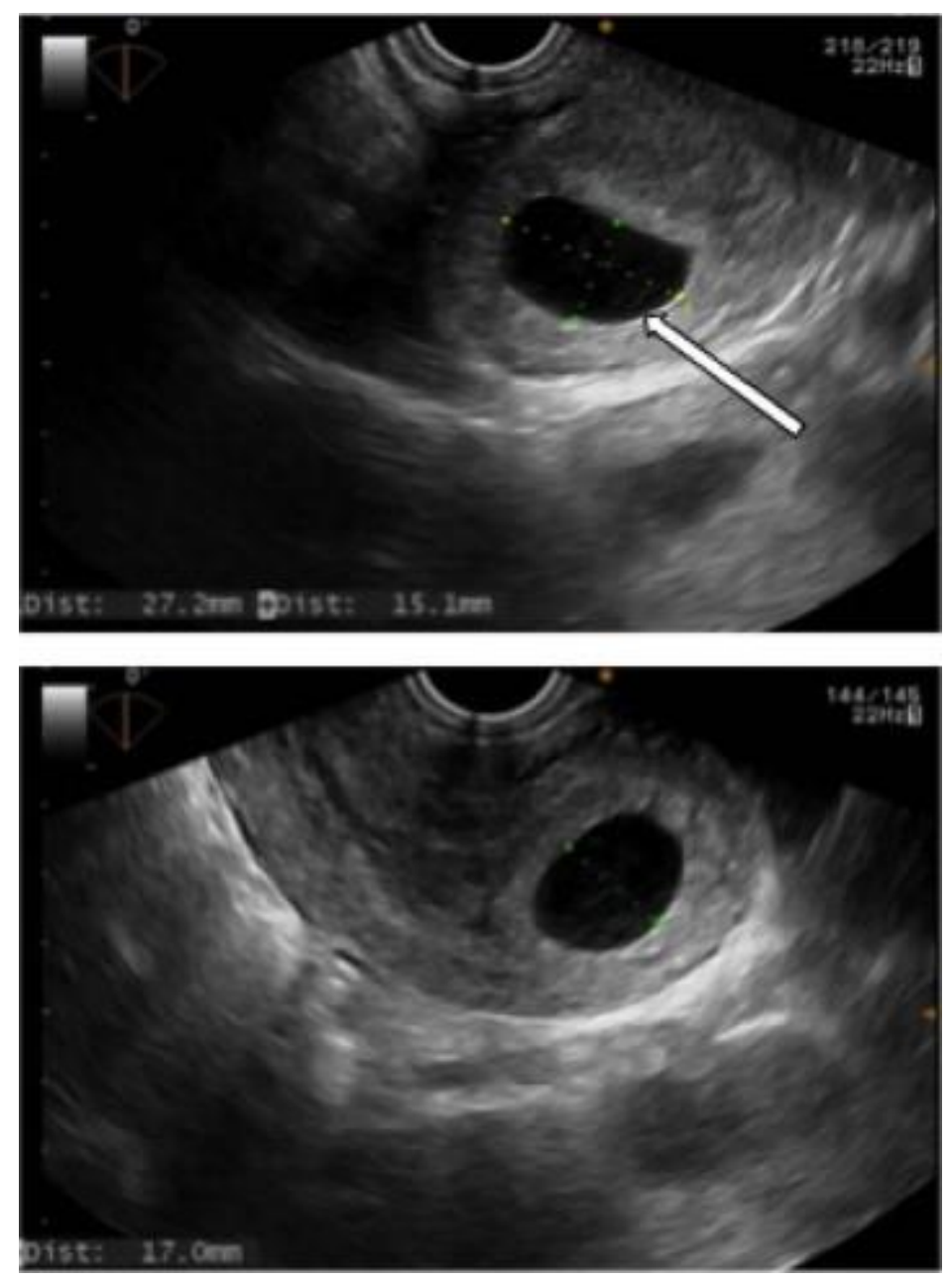

Légende figure 2: Les calipers sont positionnés à la limite externe de la zone anéchogène du sac gestationnel, soit au bord interne de la zone échogène de la couronne trophoblastique (flèche blanche). La mesure du sac gestationnel correspond à la moyenne de 3 mesures orthogonales : $(27,2+15,1+17,0) / 3=19,8 \mathrm{~mm}$, en échographie endovaginale. 
Figure 3 : Position des calipers pour la mesure du pôle embryonnaire.

Figure 3: Place of calipers for embryon crown-rump length measurement with ultrasonography

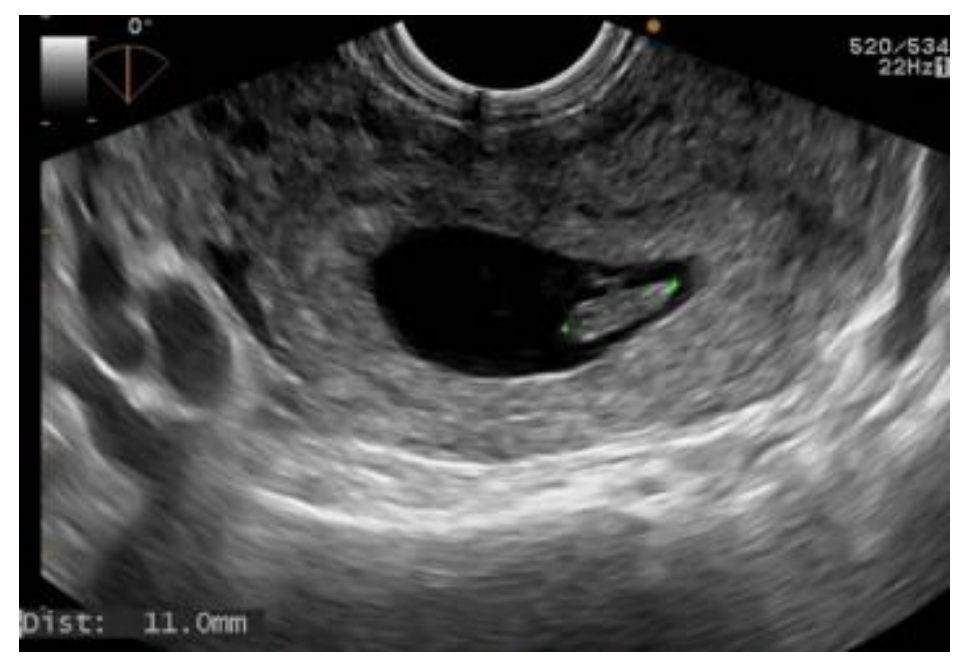

Légende figure 3 : En échographie endovaginale, la mesure embryonnaire repose sur la mesure de la longueur cranio-caudale en coupe sagittale, en plaçant les calipers de mesure sur le bord externe de la partie craniale et de la partie caudale du pôle embryonnaire. 
Figure 4 : Arbre décisionnel pour le diagnostic échographique par voie vaginale de l'évolutivité de la grossesse chez les femmes présentant une grossesse d'évolutivité incertaine.

Figure 4: Decision tree for transvaginal ultrasonographic diagnosis of pregnancy viability or failure in women with an intrauterine pregnancy of uncertain viability.

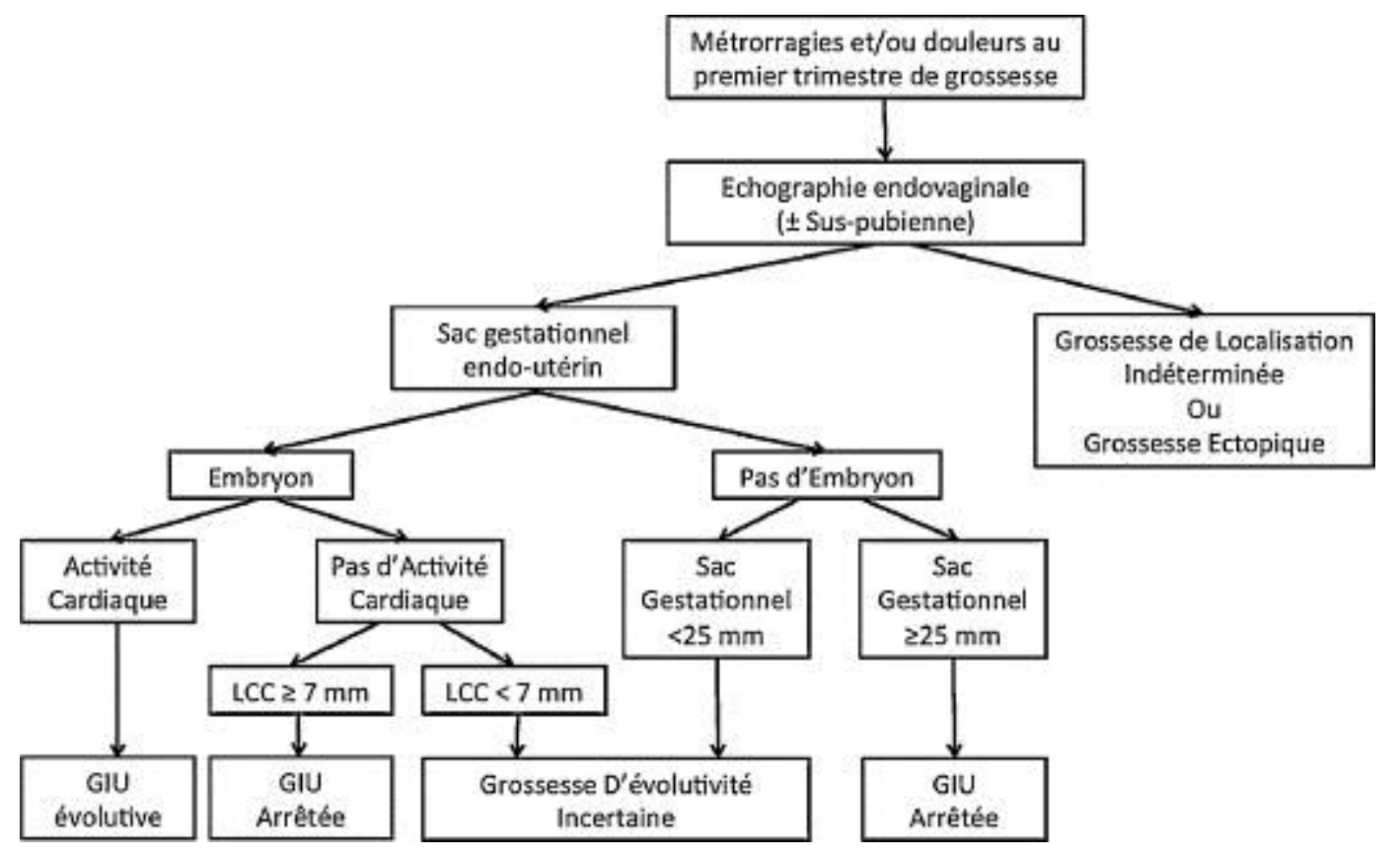


Figure 5 : Délais nécessaires pour affirmer une grossesse arrêtée en cas de grossesse d'évolutivité incertaine à l'échographie endovaginale.

Figure 5: Time-based criteria for failed pregnancy diagnosis in women with an intrauterine pregnancy of uncertain viability transvaginal ultrasonography diagnosis.

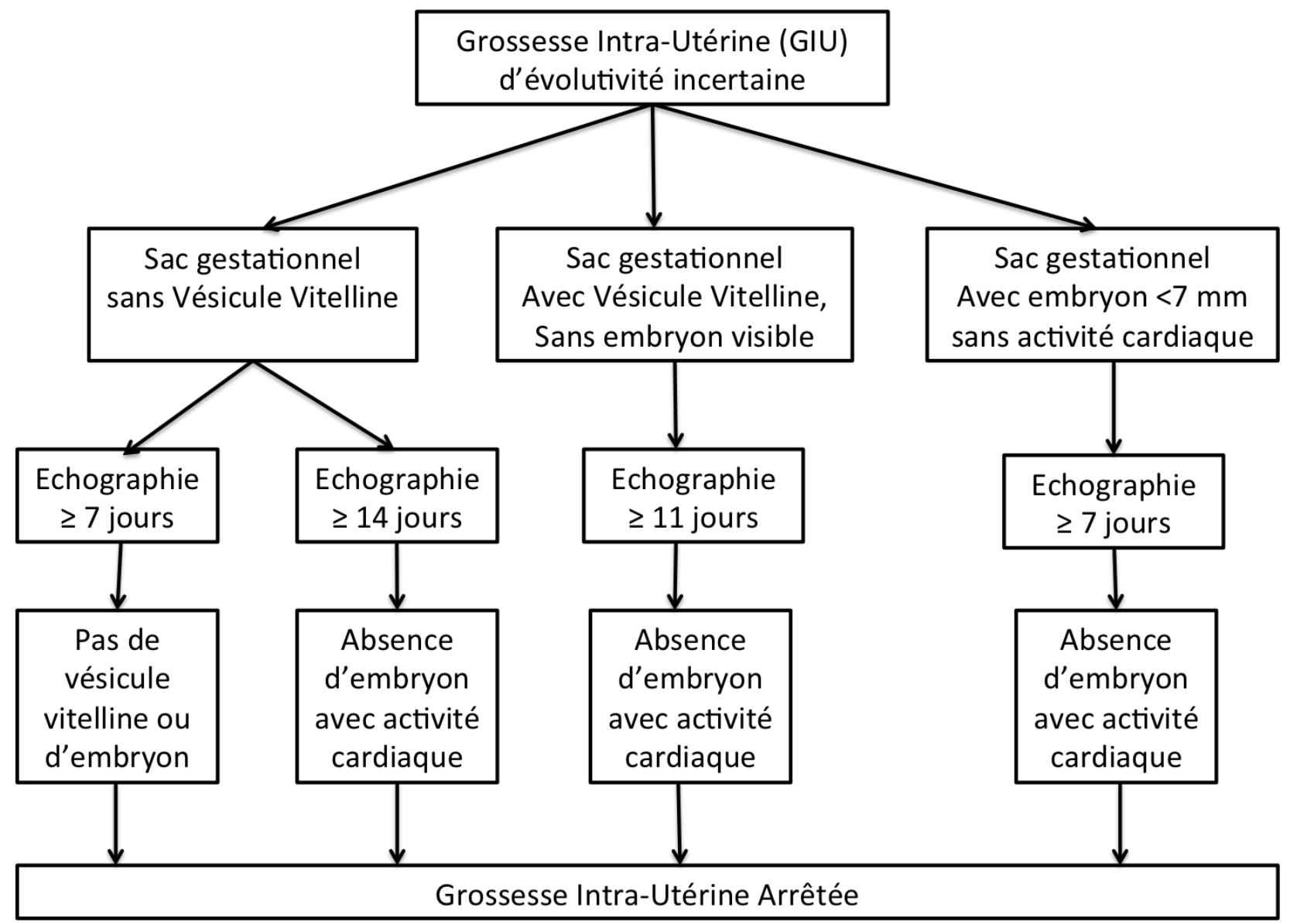


Tableau 1 : Croissance du sac gestationnel et de l'embryon en fonction du terme au premier trimestre de grossesse.

Table 1: Predicted menstrual age in weeks from gestational sac measurement and embryon crown-rump length during first trimester pregnancy

\begin{tabular}{|c|c|c|c|c|c|}
\hline $\begin{array}{l}\text { Taille du sac } \\
\text { gestationnel } \\
\text { (en mm) } \\
\text { (moyenne de } 2 \\
\text { mesures } \\
\text { orthogonales*) }\end{array}$ & $\begin{array}{l}\text { Taille de la } \\
\text { Vésicule } \\
\text { vitelline (en } \\
\text { mm) } \\
\text { (moyenne de } \\
2 \text { mesures } \\
\text { orthogonales) }\end{array}$ & $\begin{array}{l}\text { Longueur } \\
\text { cranio- } \\
\text { caudale de } \\
\text { l'embryon } \\
\text { (en mm) }\end{array}$ & $\begin{array}{l}\text { Activité } \\
\text { cardiaque }\end{array}$ & $\begin{array}{l}\text { Age } \\
\text { gestationnel } \\
\text { en Jour } \\
\text { d'Aménorrhée }\end{array}$ & $\begin{array}{l}\text { Age } \\
\text { gestationnel } \\
\text { en Semaine } \\
\text { d'Aménorrhée }\end{array}$ \\
\hline $2-5$ & - & - & & $28-31$ & \\
\hline 8 & - & - & & 34 & \\
\hline 10 & Apparition & - & & 35 & $5 \mathrm{SA}$ \\
\hline 10 & 3,0 & \multirow{4}{*}{1} & \multirow{11}{*}{$\begin{array}{l}0 \\
\frac{0}{\infty} \\
0 \\
0 \\
0\end{array}$} & 36 & \\
\hline 10 & & & & 37 & \\
\hline 11 & & & & 38 & \\
\hline 12 & & & & 39 & \\
\hline 13 & & 2 & & 40 & \\
\hline 14 & & 3 & & 41 & \\
\hline 15 & 3,0 & 4 & & 42 & $6 \mathrm{SA}$ \\
\hline 15 & & 4 & & 43 & \\
\hline 15 & & 5 & & 44 & \\
\hline 16 & & 5 & & 45 & \\
\hline 16 & & 6 & & 46 & \\
\hline 17 & & 7 & \multirow{24}{*}{ 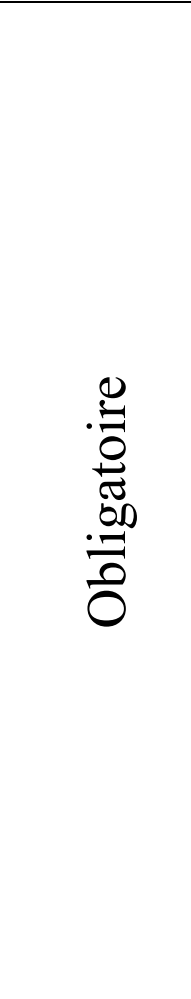 } & 47 & \\
\hline 18 & & 8 & & 48 & \\
\hline 20 & 4,0 & 9 & & 49 & $7 \mathrm{SA}$ \\
\hline 21 & & 10 & & 50 & \\
\hline 22 & & 11 & & 51 & \\
\hline 23 & & 12 & & 52 & \\
\hline 24 & & 13 & & 53 & \\
\hline 25 & & 14 & & 54 & \\
\hline 26 & & 15 & & 55 & \\
\hline 27 & 4,7 & 15 & & 56 & $8 \mathrm{SA}$ \\
\hline 29 & & 16 & & 57 & \\
\hline 30 & & 17 & & 58 & \\
\hline 32 & & 18 & & 59 & \\
\hline 34 & & 19 & & 60 & \\
\hline 36 & & 20 & & 61 & \\
\hline 37 & & 21 & & 62 & \\
\hline 38 & 5,2 & 22 & & 63 & $9 \mathrm{SA}$ \\
\hline 39 & & 23 & & 64 & \\
\hline 40 & & 24 & & 65 & \\
\hline 41 & & $25-26$ & & 66 & \\
\hline 42 & & 27 & & 67 & \\
\hline 43 & & 28 & & 68 & \\
\hline 44 & & 29 & & 69 & \\
\hline 45 & 5,8 & 30 & & 70 & $10 \mathrm{SA}$ \\
\hline
\end{tabular}

Adapté de Coulam et al [6], Wisser et al [13], Hadlock et al [11], Jauniaux et al [7], et Hellman et al [17] les distributions des mesures pour chaque terme n'ont pas été rapportées dans le tableau pour plus de lisibilité. 
* Ces courbes de croissances ont été établies par des études datant des années 80 et 90 qui prenaient la moyenne de deux mesures orthogonales du sac gestationnel. Les études publiées après les années 2000 portant sur le diagnostic d'une grossesse arrêtée ont utilisé la moyenne de 3 mesures orthogonales du sac gestationnel pour établir le seuil au-dessus duquel une grossesse sans embryon est une grossesse arrêtée.

NB : de 36 à 47 jours d'aménorrhée, la croissance embryonnaire paraît lente (moins de $1 \mathrm{~mm} /$ jour), mais il faut tenir compte de la variabilité d'apparition des structures embryonnaires (pôle embryonnaire et activité cardiaque) d'une grossesse à l'autre. Ainsi, l'embryon peut être vu à partir de 36, 37, 38 ou 39 jours mais pas pour toutes les grossesses, de même que pour l'activité cardiaque embryonnaire. 
Tableau 2 : Définitions pour le diagnostic échographique par voie vaginale de l'évolutivité de la grossesse chez les femmes présentant une grossesse d'évolutivité incertaine.

Table 2: Definitions for transvaginal ultrasonographic diagnosis of pregnancy viability or failure in women with an intrauterine pregnancy of uncertain viability.

\begin{tabular}{lc}
\hline Concept & Définition échographique \\
\hline Grossesse intra-utérine & - Embryon avec activité cardiaque visible \\
évolutive & (ne préjuge pas de l'évolution, un arrêt de grossesse peut \\
avoir lieu dans le suivi ultérieur)
\end{tabular}

Grossesse intra-utérine arrêtée

Grossesse intra-utérine d'évolutivité incertaine

Grossesse de localisation indéterminée

Grossesse ectopique
- Sac gestationnel intra-utérin $\geq 25 \mathrm{~mm}$ (moyenne des 3 mesures orthogonales du sac gestationnel par échographie endovaginale) sans embryon

- Embryon intra-utérin $\geq 7 \mathrm{~mm}$ sans activité cardiaque (mesure sagittale cranio-caudale de l'embryon par voie échographique endovaginale)

- Grossesse intra-utérine qui n'est ni une GIU évolutive, ni une GIU arrêtée.

- Sac gestationnel intra-utérin $<25 \mathrm{~mm}$ (moyenne des 3 mesures orthogonales du sac gestationnel par échographie endovaginale) sans embryon

- Embryon intra-utérin $<7 \mathrm{~mm}$ sans activité cardiaque (mesure sagittale cranio-caudale de l'embryon par voie échographique endovaginale)

- Absence de visualisation de sac gestationnel chez une femme enceinte (hCG positif urinaire ou sanguin)

- Sac gestationnel, avec ou sans embryon, visualisé en dehors de la cavité utérine

GIU : Grossesse intra-utérine.

Ces définitions doivent figurer dans la conclusion du compte-rendu d'échographie réalisée pour une grossesse au premier trimestre de grossesse. 